\title{
Introduction to Optimal and Robust Control
}

\subsection{Introduction}

There have been at least five distinct stages in the development of the subject of modelbased control systems. Early work by Nyquist [1], Bode [2] and Nichols [3] was concerned with frequency-response design methods for single-input single-output (scalar) systems. The importance of poles and zeros became evident early in the development of the subject and the root locus technique was developed by Evans in 1948 [4]. Gain and phase margins were used widely as measures of the quality of design and gave an indication of stability robustness. Rules of thumb were and are still used in classical design. For example, it was found that reasonable closed-loop time responses were often obtained with gain margins in the range $2-5$ and phase margins in the range $30-60^{\circ}$. The Bode design technique was found to have inherent robustness properties, even though these were not emphasised until the later work of Horowitz [5].

The frequency response approach to optimal control and filtering, introduced by Norbert Wiener [6], was not necessarily robust. The approach concentrated on the minimisation of the variance of signals rather than the minimisation of functions which determined robustness properties. The design method did not by construction ensure asymptotic stability of the closed-loop system. Unstable pole-zero cancellation, between the controller and plant, could therefore occur. The resulting system is of course unstable in such a case.

The space race during the 1960 s provided the impetus for the strong growth of socalled modern control theory. The Soviet Union's success in launching the Sputnik spacecraft in the late 1950s prompted President Kennedy's pledge in the early 1960s to land a human on the moon and this stimulated the development of new control design methods. There were of course many other influences and needs from all sectors of industry, which helped the development of new control techniques, but optimal control and estimation were particularly encouraged by the applications in space. The most significant contributions were by Kalman [7] who introduced the state-space approach to optimal control and filtering theory. In this second stage of development the system models were assumed to be known exactly (like the Wiener solution procedures). Some inherent robustness properties [8] were obtained quite naturally for state feedback solutions, but not for the important case of output feedback systems.

Robust Industrial Control Systems-Optimal Design Approach for Polynomial Systems M. J. Grimble (C) 2006 John Wiley \& Sons, Ltd 
In the third stage of control theory development, attention turned to the design of multivariable systems by frequency domain design methods. Thus, extensions of the scalar classical frequency-response techniques for multi-input multi-output (MIMO) systems were developed by Rosenbrock [9], MacFarlane [10] and Mayne [11]. Although Rosenbrock considered high-integrity designs that could cope with loop gain degradation, these techniques did not provide formal methods of improving robustness in the face of modelling uncertainties.

The fourth stage in the design of systems was concerned with producing robust design procedures and most of the attention was involved with the so-called $H_{\infty}$ design philosophy. The approach by Zames [1,12] allowed robustness to be dealt with far more directly than with other optimisation procedures. The work considered some of the basic questions of classical control theory and attracted immediate attention. Robustness was of course always an issue in design, but Zames knew that a more scientific approach was needed for the design of uncertain systems. This method has enabled engineers to exploit classical frequency response design insights using a technique which can be implemented in computer-aided control system design environments. Thus, uncertainties were allowed for in a rigorous design framework with the advantages of classical design insights and a formal design procedure. The quantitative feedback theory of Horowitz also provided a route to robust control design that was particularly successful with those who liked the nonoptimal classically based design stages. This methodology is simple to use for single-input single-output (SISO) and it provides an understanding of the real issues of uncertainty that is often lacking in $H_{\infty}$-based methods. Unfortunately it is difficult to use formal standardised design procedures, particularly for the multi-input multi-output (MIMO) case, but it does involve a strong and committed user community.

The fifth and current stage of development lies in the successful application of modern control methods, which is closely related to problems in nonlinear control systems design. Significant progress has been made in the development of optimal control techniques for nonlinear systems and in performance assessment and benchmarking methods for real applications. The next decade should see continuing developments in the application of advanced techniques to the most challenging uncertain, multivariable and nonlinear systems, where improved control can provide significant performance or economic advantages. For example, modern fighter aircraft undertake manoeuvres well outside the linear flight dynamics, in order to achieve tactical advantages. Nonlinear control is therefore essential in certain applications. There have been many applications of predictive optimal control or so-called model-based predictive control methods in real applications and nonlinear versions are currently under development for process and manufacturing system applications. There is also developing interest in the control of socalled hybrid systems, which can involve a combination of finite state machines and dynamical systems. A typical hybrid control problem arises in gas turbine control startup or shut-down sequences, where a mixture of logical decision making and continuous control is required.

\subsubsection{Optimality, Feedback and Robustness}

There has been a long history in the development of analytical methods for the determination of optimal control policies for linear and nonlinear systems subject to a 
given performance index. Euler and Lagrange are often thought to be the founders of variational theory about 180 years ago. The calculus of variations is concerned with the maximisation or minimisation of areas and surfaces in multi-dimensional space and both Newton and Leibnitz were interested in such variational problems. The simplest class of optimisation problem is considered in most of the following concerning linear timeinvariant systems.

The optimal control paradigm followed below involves the minimisation of a cost function, usually of energy $\left(H_{2}\right)$ or peak frequency-response $\left(H_{\infty}\right)$ form. However, the cost function is often only used as a means of specifying the design requirements and providing the very important tuning variables. Optimality may not be so important. In many cases it only provides the mechanism by which good feedback control designs can be achieved, where the measure of goodness can be in terms of classical stability criteria and robustness.

Certain properties of a system can be changed by feedback, but not by open-loop compensation. Feedback properties include stability, stability margins and disturbance rejection and these can be altered only by the use of feedback. Robustness is normally improved by the use of feedback, and a robust design gives large stability margins, good disturbance attenuation and/or low sensitivity.

The response of a system to commands is not a feedback property of a system, although it is often treated as such. The command response of a system can be modified without the use of feedback, using an open loop precompensator. Stability margins provide an important characterisation of system properties. They can provide a lower bound on the magnitude (in some sense) of the smallest perturbation to the loop gain or return-ratio matrix, which will destabilise the system.

The term modern control was introduced in an era that is usually believed to span the period 1960-1980. This coincided with the introduction of state-space-based synthesis techniques and included the period when LQ optimal control techniques became very popular. These optimal control solutions were normally thought of as being time-domain methods; although Parseval's theorem enabled the optimal control problem to be posed equally well in the frequency domain [16]. Towards the very end of the modern control era, Kucera [17] published his seminal work on the use of polynomial-based methods in the solution of optimal control problems. It was this work, and the developments on $H_{\infty}$ control by Kwakernaak [18] which inspired much of the following text. However, the notation employed is closer to that used in the adaptive control or self-tuning literature and it was this type of application which originally motivated interest in the polynomial systems approach.

The mathematical framework employed in the following polynomial analysis is a little different from that of Kucera [17]. The analytical framework employs $H_{2}$ optimisation, where $\mathrm{H}_{2}$ represents the Hardy space of functions analytic in $|z|<1$ that are squareintegrable on $|z|=1$. The mathematical basis of polynomial systems theory was discussed in Barnett [33], Wolovich [34], Kailath [35] and Vardulakis [77].

\subsubsection{High-integrity and Fault-tolerant Control Systems}

There are some applications where a high-integrity design is required. This is the property whereby the closed-loop remains stable against arbitrary feedback-loop failures. 
That is, the control system remains stable, even when the gain in a certain loop or loops is reduced, possibly to zero. For example, when a feedback signal is accidentally broken it is desirable that this should not cause instability. The linear quadratic $(L Q)$ state or output feedback optimal regulator does not, under standard conditions, guarantee stability in such a case, even when the plant is open-loop stable. A similar problem arises in situations where the actuators are prone to saturation. In this case the gain effectively reduces in the loop when the system is in saturation, which can also lead to instability.

The inverse Nyquist array (INA) design technique is one of the few multi-variable design procedures, which enables a high integrity design to be achieved in a natural manner [9]. However, modifications to LQ design procedures have also been proposed to enable improvements in integrity to be obtained. The high-integrity design problem has not been analysed to the same extent using $H_{\infty}$ design procedures, although gain reduction tolerance can be built in by introducing an uncertainty model. Loop failures may be particularly important in some safety critical applications.

The whole area of fault-tolerant control has great importance for many applications, ranging from aerospace to automotive systems. One of the areas for future development is the so-called self-healing approach, which involves a combination of ideas from intelligent control and model-based learning control systems. It is of course an ideal to have a control system, which recognises the build-up of fault conditions in actuators or sensors, and then takes corrective action. This suggests the importance of self-monitoring possibly using data-driven condition monitoring methods. Significant computing power is needed to provide such facilities in distributed embedded systems.

\subsubsection{Self-healing Control Systems}

The self-healing control philosophy is meant to suggest something rather more than traditional methods of fault-tolerant control or reliable control. The self-healing concept involves ideas from learning systems where intelligence is built into the control law, in addition to the usual algorithmic tuning features. Inherent in the self-healing idea is the use of self-monitoring, which refers to controller monitoring rather than process condition monitoring. In such a system the performance of the controller is assessed periodically and self-healing action is provided based on a combination of previous intelligence and information.

Although learning systems can be applied to any controller structure, one of the most promising approaches is to use restricted structure $(R S)$ controllers, where the tuning variables are necessarily restricted and the controller has a traditional simple structure. Not only is this more appealing to engineers in industry, but it also limits the range of tuning options available. Such controllers have been shown to be useful for nonlinear systems, based upon a multiple linear model design concept. The performance deterioration, relative to a full-order design, is often a lot less than would be expected. The controller to be implemented has a simple structure in this case and is simple to understand and to tune.

The self-healing action involves computations in the background that are part of the supervisory system. Since the controller parameters are adjusted only in the case of a build-up of fault conditions, they are not continuously adjusted, as in the case of self 
tuning control. Thus, for most of its working life the controller acts like any other optimal or classically designed controller, but, when fault conditions start to arise, decisions are made as to the actions to be taken and warnings are given to process management and technical staff via communication links. Whilst the self-healing controller is compensating for problems in sensors or actuators, its own performance is monitored to ensure stability of the system is maintained and risks are not taken. To gather information in this phase, additional probing test signals may be used and logical decisions are then taken based on reasoning and on historical information.

The self-healing controller should be of value in safety-critical industries and in all areas where continuity of production is of paramount importance. The software for such systems could be in local controllers or in supervisory control and data acquisition (SCADA) systems.

\subsubsection{Fault Monitoring and Detection}

Condition and fault monitoring control systems are as important as the design of the control loops. Great strides have been made in the development of sensors and in new wireless systems, which provide distributed condition monitoring facilities with redundancy. These developments may well revolutionise the control problem, since even a scalar feedback loop may become a single-input multi-output system when low-cost sensors with wireless communications are readily available. There have been many advances in the development of model-based fault monitoring and diagnosis systems [84]. For example, the $H_{\infty}$ estimation techniques are important for uncertain systems and are now finding applications in fault detection and monitoring systems $[71,72]$. The $H_{\infty}$ filter is valuable for systems where the estimation error, in a particular frequency range, should be limited, and it can also be chosen to be optimal when the system model is uncertain. The $H_{\infty}$ filter has considerable potential in applications such as condition monitoring and fault detection.

\subsubsection{Adaptive versus Robust Control}

There are two approaches to allow for uncertainty in system models and disturbances. The first approach is to use an adaptive controller, which estimates parameters and calculates the control accordingly. Self-tuning devices have been very successful, but they involve on-line design computations and are therefore not as simple as a fixed controller to implement. The second approach is to allow for uncertainty in the design of the fixed controller, thus producing a so-called robust control scheme, that is, one which is insensitive to parameter variations or disturbances. The $H_{\infty}$ robust control design philosophy provides an optimal approach to improve the robustness of a controlled system. The $H_{\infty}$ design approach can be combined with self-tuning action to obtain a socalled robust adaptive controller [14, 15, 32].

\subsubsection{Artificial Intelligence, Neural Networks and Fuzzy Control}

In the 1940s Norbert Wiener (1894-1964) introduced the term cybernetics to describe the related subjects of control and communications in humans and machines. In the 1960s 
analytical techniques were particularly successful, but in, for example, the aerospace industry cybernetics did not seem particularly relevant. This type of approach became more important in the chemical, petrochemical and pharmaceutical industries. The major intelligent control (IC) approaches include: fuzzy logic, artificial neural networks, genetic algorithm, expert systems and fuzzy-neural systems. The search for new paradigms to solve problems in robotics and computer vision in the 1980s stimulated activity in artificial neural networks (ANNs) and this resulted in renewed interest in the subject of cybernetics. Intelligence is now needed in control and instrumentation systems, at both regulating loop and supervisory levels. It is therefore likely that the model-based analysis and synthesis techniques to be presented will be enhanced by these alternative technologies, providing learning and decision making capabilities, which can significantly improve capabilities [62].

Identification of nonlinearities is often needed for both fixed and adaptive control design. A universal function for approximation is therefore needed that can, for example, approximate an unknown static nonlinear function representing system actuators. There are various neural and fuzzy approaches that may be employed. Neural networks provide techniques for implementing identification and optimisation algorithms that may have an important role in the control of unknown nonlinear systems.

The theoretical foundations of fuzzy logic were developed by Lofti A. Zadeh, using the concept of fuzzy sets, which are a generalisation of conventional set theory. Fuzzy sets are based on ideas from multivalued logic, probability theory, artificial intelligence and artificial neural networks. Fuzzy logic does not classify things as true or false and does not draw conclusions from exact numerical values. It might be considered an extension to normal Boolean logic and Fuzzy logic theory is based on extending Boolean values to include all real values between 0 and 1 . This may be used to classify arbitrary measured values from, say, process control measurements. Before the fuzzy rules can be applied to direct process measurements, a fuzzification operation is needed. This function can be used to define membership set values in a range between 0 and 1 .

Fuzzy controllers are implemented using fuzzy rules, which can reduce the number of computations in conventional controllers. It is also claimed that they can be implemented more easily than conventional controllers. The most popular kind of fuzzy systems are based on either the Mamdami fuzzy model, Takagi-Sugeno-Kang (TSK) fuzzy model, Tsukamoto fuzzy model or Singleton fuzzy model. To define a fuzzy logic controller it is necessary to introduce if-then rules to establish how probable it is that a process variable, such as a level is high or a temperature is cold. To evaluate the rules, the definition of fuzzy operations is also needed. The application of the rules, defines fuzzy set values of fuzzy output sets. The probabilities of the open/close control actions for a valve can be related directly to fuzzy set (membership) values taken between 0 and 1 .

The inverse operation to fuzzification is referred to as defuzzification. This function is used to interpret the fuzzy set values of open/close control actions to provide real numbers defining say actual valve positions on a scale of say 0 to 1 , and there are many different methods, such as the Sugeno method. Unfortunately the internal structure of a fuzzy logic controller is not so clear and to some extent fuzzy logic tools hide the internal structure from the designer. An advantage of the approach is that only a basic understanding is needed to use fuzzy logic as a control strategy. Fuzzy logic is also well suited to processes that are difficult to describe by means of a conventional process model, but 
are based on manual control experience. There are many recent applications of fuzzy systems. The most famous application is for the Sendai Underground in Japan. Fuzzy systems also include a fuzzy structure mixed with other kinds of approach (optimal control, robust control, adaptive). Fuzzy logic is often claimed to be a useful tool in cases where the process involves strongly nonlinear features.

Neuro-fuzzy modelling has been suggested for process modelling and control due to the robustness and fault tolerance required in industrial applications [98]. The technique may be applied to systems with high degrees of uncertainty, or to systems without a model, or including time-varying and nonlinear characteristics $[99,100]$. The technique makes it easy to develop models using a combination of empirical models, heuristics and real data. A neuro-fuzzy model is a type of hybrid fuzzy system which is represented by fuzzy if-then rules in the neural network. Neuro-fuzzy systems can be interpreted and analyzed as a fuzzy system and trained as a neural network. Neuro-fuzzy modelling uses local linear models in order to represent the full operating regime. It involves a decomposition of the nonlinear plant so that each operation range is modelled by a local linear model [101]. Fuzzy membership functions may be defined as the weighted combination of the local models in order to approximate the nonlinear behaviour of the plant. A decision is taken on which model or combination of models is active and appropriate in a certain operating regime of the plant. This is one of the most popular techniques used in nonlinear system identification.

Expert systems and knowledge-based systems are also valuable for management information systems, and are particularly successful in the metal processing industries $[66,67]$. Knowledge-based reasoning is one of the tools that may be used in self-healing controllers.

\subsubsection{Discrete-time Systems}

A digital control system normally involves a process which in some part is in digital form. Sampled data control systems are very common, normally involving a continuous process with computer control. The implementation of digital controllers can involve programming digital signal processors (DSPS). A typical sampled-data system will involve a continuous reference signal that is sampled using an analog-to-digital converter $(A D C)$. A digital controller usually operates on the difference of the sampled reference and output signals. The output of the digital controller will then feed a digital-to-analog converter (DAC). The measured plant output can be sampled to enable the set-point following error to be computed. Digital systems are simple to modify and update and they can be used for multiple tasks. There are some problems introduced by the sampling process, including the need to choose a sampling rate and the difficulties of scaling and round-off errors, but these are not very significant relative to the advantages.

For an effective DSP system the sampling rate must be at least twice the highest frequency component in a signal (from the sampling theorem). If this rule is not satisfied, information from the continuous process will be lost by the sampling operation. The original signal cannot then be reconstructed without introducing distortion. If the sampling occurs at less than this rate, aliasing problems will arise. Aliasing involves the overlapping of spectra and introduction of noise in the signal pass-band. For a control 
system the sampling rate might be selected to be about ten times the system's bandwidth [83].

Notation: The notation employed in the following for the discrete-time system models, is introduced in the Appendix, which also includes an explanation of many of the basic mathematical results that are needed. The transfer function or polynomial matrices to be defined are functions of the indeterminate $z^{-1}$. In the complex domain optimisation analysis $z$ represents the $z$-transform complex number. However, following common usage $z^{-1}$ will also denote the unit-delay operator, in the time-domain form of the system equations. It should be clear from the context of its use, which interpretation is appropriate. To simplify notation the dependence of the transfer functions on $z^{-1}$ may not be shown explicitly.

\subsection{The $H_{2}$ and $H_{\infty}$ Spaces and Norms}

The theory of Hardy $\left(H_{P}\right)$ spaces has its origins in discoveries made about 90 years ago by mathematicians including: G.H. Hardy, J.E. Littlewood, I.I. Privalov, F. and M. Riesz, V. Smirnov and G. Szego. An introduction to this class of spaces was given by Duren [19]. Renewed interest in the $H_{P}$ spaces occurred in the 1970s with the development of the subject of functional analysis and new applications have arisen in recent years, particularly in systems science. The historical starting point for the theory of $H_{P}$ spaces is the 1915 paper by Hardy [20], dealing with Hardy's convexity theorem.

The $H_{\infty}$ robust design approach to control systems design was motivated and stimulated by the work of Zames [13] and Zames and Francis [21] in 1981. Zames argued that the mathematical framework for linear quadratic $(L Q)$ or linear quadratic gaussian $(L Q G)$ optimal control did not enable uncertain systems to be treated rigorously. He proposed a new optimisation problem in the $H_{\infty}$ space that was more suitable for systems with uncertainty. The approach was designed for systems with unmodelled dynamics in the plant or in the disturbance models. He was able to show that the family of $L Q$ control laws, which can be related to the solution of problems in the $\mathrm{H}_{2}$ space, were not robust to different forms of uncertainty. That is, the traditional least-squares optimisation problems were equivalent to minimising a $\mathrm{H}_{2}$ norm and in some cases do not provide robust solutions.

The $H_{\infty}$ space is the space of transfer functions of causal stable linear time-invariant systems. Such systems have bounded input bounded output (BIBO) stability for squareintegrable $\left(L_{2}\right)$ inputs and outputs. In the scalar case, the $H_{\infty}$ norm (denoted $\|\cdot\|_{\infty}$ ) is simply the maximum amplitude of the frequency response of the transfer function. Thus, the $H_{\infty}$ norm represents an important measure of gain, often used in classical design for measuring the peaks of sensitivity or closed-loop transfer functions. A consequence is that the following norm relationship applies: $\left\|W\left(z^{-1}\right) G\left(z^{-1}\right)\right\|_{\infty} \leq\left\|W\left(z^{-1}\right)\right\|_{\infty}$ $\left\|G\left(z^{-1}\right)\right\|_{\infty}$ where the transfers: $W\left(z^{-1}\right)$ and $G\left(z^{-1}\right)$ are $z$-transfer-function matrices. Unfortunately the quadratic norms used in $H_{2} / L Q G$ design do not satisfy this multiplicative relationship, which is needed for uncertain systems that include norm-bounded uncertainty $[13,21]$. Uncertainty is the main reason that the $H_{\infty}$ norm is so important in the robust design of control systems. Note that whenever a perturbation analysis is used, or when plant modelling errors are considered, products or cascaded transfer functions occur. If bounds on the magnitudes of the resulting system transfer function 


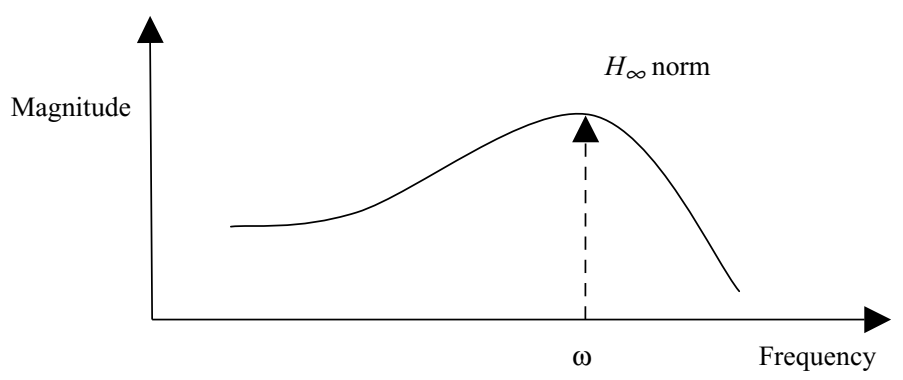

Figure 1.1 $H_{\infty}$ norm of scalar system obtained from maximum on Bode diagram

are to be obtained, a multiplicative norm relationship is needed. The $H_{\infty}$ optimisation approach is therefore valuable for the robust control design of some classes of uncertain systems.

\subsubsection{Graphical Interpretation of the $\mathrm{H}_{\infty}$ Norm}

The $H_{\infty}$ norm of a certain input-output transfer is a measure of the gain of the system. It can be represented by the maximum value of the ratio of the output and input energy of the respective signals. However, it is easier to understand the physical significance of the $H_{\infty}$ norm by looking at the frequency-response magnitude of a scalar transfer function, shown in the Bode magnitude diagram of Figure 1.1.

The maximum amplitude of the frequency response, or maximum gain, is equal to the $H_{\infty}$ norm in the scalar case. When robustness is to be improved there is often the need to limit the gain in a particular frequency range and this can be achieved by minimising some (weighted) transfer function. The weighting can be achieved by simply multiplying the transfer of interest by a weighting filter of suitable frequency response.

\subsubsection{Terms Used in $H_{\infty}$ Robust Control Systems Design}

The following summary of terms used in $H_{\infty}$ synthesis and design problems may be a useful reference source for the material in the remainder of the chapter. In most cases more precise mathematical definitions are presented at the point of use, or in the Appendix. The summary follows in Table 1.1.

\subsection{Introduction to $H_{\infty}$ Control Design}

Robust control systems design is one of the most important areas for industrial control applications [78], that can take into account errors in modelling a system. A plant may contain both structured and unstructured uncertainty. For example, a plant transfer function: $W\left(z^{-1}\right)$ with structured uncertainty might have the form: $W\left(z^{-1}\right)=z^{-2}$ / $\left(1+\alpha z^{-1}+\beta z^{-2}\right)$, where $\alpha$ and $\beta$ belong to some known intervals $\alpha \epsilon\left[\alpha_{1}, \alpha_{2}\right]$ and $\beta \epsilon\left[\beta_{1}, \beta_{2}\right]$, respectively. The $H_{\infty}$ approach to optimal control system design is particularly appropriate for the stabilisation of plants with unstructured uncertainty. Unstructured uncertainty can represent modelling errors in a system, where there is no information 
Table 1.1 Terminology of uncertain systems and robust control

\begin{tabular}{|c|c|}
\hline$H_{\infty}$ norm & $\begin{array}{l}\text { A measure of the size of a transfer function that may be estimated by } \\
\text { plotting the Bode amplitude diagram and noting the maximum } \\
\text { value of the frequency response }\end{array}$ \\
\hline $\begin{array}{l}\text { Stability } \\
\quad \text { robustness }\end{array}$ & $\begin{array}{l}\text { The ability of the system to remain stable, even though the model used } \\
\text { for system design is very different from the plant model which exists } \\
\text { in practice }\end{array}$ \\
\hline $\begin{array}{l}\text { Performance } \\
\text { robustness }\end{array}$ & $\begin{array}{l}\text { Ability of the system to maintain good performance, measured in } \\
\text { terms of its tracking accuracy, given that modelling errors exist } \\
\text { when designing the controller }\end{array}$ \\
\hline Singular value & $\begin{array}{l}\text { Provides a measure of the size of a matrix and is similar to an } \\
\text { eigenvalue, but is more reliable numerically }\end{array}$ \\
\hline $\begin{array}{l}\text { Structured } \\
\quad \text { singular value }\end{array}$ & $\begin{array}{l}\text { Similar to the singular value, but enables the structure of the } \\
\text { uncertainty to be taken into account so that less conservative designs } \\
\text { can be achieved }\end{array}$ \\
\hline Hardy space & $\begin{array}{l}\text { Mathematical setting in which } H_{\infty} \text { optimisation work is posed and is a } \\
\text { space of all stable transfer functions }\end{array}$ \\
\hline $\begin{array}{l}\text { Parametric } \\
\text { uncertainty }\end{array}$ & $\begin{array}{l}\text { The uncertainty in the definition of gains or time constants in transfer } \\
\text { functions that are otherwise well defined }\end{array}$ \\
\hline $\begin{array}{c}\text { Unmodelled } \\
\text { dynamics }\end{array}$ & $\begin{array}{l}\text { Represents part of the system transfer function or state model } \\
\text { which is neglected or unknown when basing a design on a nominal } \\
\text { plant model (usually low order) }\end{array}$ \\
\hline $\begin{array}{l}\text { Unstructured } \\
\text { uncertainty }\end{array}$ & $\begin{array}{l}\text { May be an unrealistic representation of uncertainty, since it allows for } \\
\text { the presence of modelling errors in all of the elements of a system } \\
\text { transfer function, whereas modelling errors may only be possible } \\
\text { of a well-defined structure }\end{array}$ \\
\hline$\mu$ synthesis & $\begin{array}{l}\text { This is a design method which uses repeated iterations of a } H_{\infty} \text { design } \\
\text { algorithm and invokes the structured singular value to test if the design } \\
\text { is robust }\end{array}$ \\
\hline
\end{tabular}

available except for an upper bound on its magnitude which may be a function of frequency.

Unstructured uncertainty models are used in the following analysis, since general $H_{\infty}$ techniques can be proposed to design robust controllers. Most system models will include unstructured uncertainty, particularly at high frequencies, since there will normally exist some unmodelled dynamics. In fact there are often good reasons why low-order models should be used for control systems design, even though the plant to be controlled is known to include high-order and possibly high-frequency, dynamics. As noted in the previous definitions, the $H_{\infty}$ norm of a scalar transfer-function is simply the maximum amplitude of the frequency response [39]. The $H_{\infty}$ normed linear space is the space of complex-valued functions of a complex variable, analytic and bounded outside the unit disc in the $z$-plane (or for continuous-time systems bounded in the right half-plane).

To consider how the $H_{\infty}$ approach can aid robust control systems design assume that a controller has been designed for a nominal system and that the open-loop transfer function is denoted by $G\left(z^{-1}\right)$. The actual system will differ from the nominal $G\left(z^{-1}\right)$ by a modelling error, which in the case of additive uncertainty would result in an open-loop transfer function: $G\left(z^{-1}\right)+\Delta G\left(z^{-1}\right)$. The question arises whether the system will remain stable given this uncertainty. Before answering this question, the uncertainty must be quantified in some way and in the $H_{\infty}$ design approach an upper bound on its magnitude, 
over all frequencies, is assumed to be known. That is, $\left|\Delta G\left(z^{-1}\right)\right|<\left|\delta G\left(z^{-1}\right)\right|$ for all $z=e^{j \omega T_{s}}$, where $\delta G\left(z^{-1}\right)$ denotes the given bounding function and $T_{s}$ represents the sampling interval for the digital control system. Recall for discrete-time systems the frequency response of a transfer function is obtained by letting: $z=e^{j \omega T_{s}}$, where the sample period $T_{s}$ is often normalised to unity for simplicity. The system may then be designed to remain stable for all perturbations, which satisfy the perturbation bound: $\left\|\delta G\left(z^{-1}\right) S\left(z^{-1}\right)\right\|_{\infty}<1$, where $S\left(z^{-1}\right)$ denotes the sensitivity function for the unityfeedback scalar system, defined as: $S\left(z^{-1}\right)=1 /\left(1+G\left(z^{-1}\right)\right)$.

Not only does this result provide a means of checking that the design will be stabilising in the presence of uncertainty, it also suggests that the maximum stability margin will be achieved when the controller is chosen to make the $H_{\infty}$ norm of the weighted sensitivity function $\left\|\delta G\left(z^{-1}\right) S\left(z^{-1}\right)\right\|_{\infty}$ small. Clearly if the sensitivity function is small, the amount of uncertainty (of the above type) that can be tolerated is increased. In the case of different types of uncertainty other frequency response functions must be minimised, such as the complementary sensitivity function, defined in the scalar case as: $T\left(z^{-1}\right)=G\left(z^{-1}\right) /\left(1+G\left(z^{-1}\right)\right)$. The performance of a system can often be optimised by minimising related frequency response functions. A typical mixed sensitivity cost function to be optimised includes both weighted sensitivity and complementary sensitivity terms. Similar results apply in multivariable systems, where the $H_{\infty}$ norm is defined as: $\|G\|_{\infty}=\sup _{\omega}\left\{\sigma_{\max }\left(G\left(e^{-j \omega T_{s}}\right)\right)\right\}$ and $\sigma_{\max }$ denotes the maximum singular value of the matrix function (see the Appendix).

\subsubsection{Properties of $H_{\infty}$ Robust Control Design}

There are several advantages of the $H_{\infty}$ control design approach. The technique can be easily computerised and formalised design procedures can be introduced. Design issues can be considered in the frequency domain and classical design intuition can be employed. However, the most important advantage is that stability margins can be guaranteed and performance requirements can also be satisfied, in a unified design framework. The main disadvantage is that methods of handling parametric uncertainty are not handled so naturally in the $H_{\infty}$ framework. A high-performance robust design would take account of this structure, but the basic $H_{\infty}$ approach does not account for this type of information. However, there are several ways of modifying the method to allow for parametric uncertainties, including $\mu$ synthesis and $H_{\infty}$ adaptive control. The $H_{\infty}$ design approach is a strong contender to provide a general purpose control design procedure, which can account for uncertainties and is simple to use with computer-aided design tools.

The $H_{\infty}$ design approach is distinguished by the following features and properties:

- It is a design procedure developed specifically to allow for the modelling errors, which are inevitable and limit high-performance control systems design.

- There is a rigorous mathematical basis for the design algorithms, which enables stability and robustness properties to be predicted with some certainty.

- The $H_{\infty}$ technique can enable classical frequency design intuition to be invoked, since most of the previous applications work has been for linear time-invariant systems. 
- There are close similarities between state-space versions of $H_{\infty}$ controllers and the well-known Kalman filtering or $\mathrm{H}_{2} / L Q G$ control structures.

- If the uncertainty lies within the class considered, stability properties can be guaranteed and safe reliable systems can be assured. Note that the design procedures cannot be used blindly, since poor information can still lead to controllers with poor performance properties.

- The trade-offs between good stability properties and good performance are easier to make in a $H_{\infty}$ context than with many of the competing designs.

- The approach can be interpreted in terms of the stochastic nature of the system, but if disturbances and noise are important $H_{2} / L Q G$ may still be the preferred solution.

- The $H_{\infty}$ design technique is easy to use, since the algorithms are available in commercial software.

\subsubsection{Comparison of $\mathrm{H}_{\infty}$ and $\mathrm{H}_{2} / L Q G$ Controllers}

The similarities and differences between the $H_{\infty}$ and the $H_{2} / L Q G$ approaches are detailed below:

\section{Similarities}

(a) Both $H_{2}$ and $H_{\infty}$ optimal controllers are based on the minimisation of a cost index.

(b) Some of the closed-loop poles of the $L G Q$ solution will be the same as those of the $H_{\infty}$ solution in certain limiting cases.

(c) The dynamic cost weights have a similar effect in both types of cost function, e.g. integral action can be introduced via an integrator in the error weighting term in both cases.

(d) Closed-loop stability can be guaranteed, whether the plant be non-minimum phase, or unstable (neglecting for the moment uncertainty and assuming controllers are implemented in full).

\section{Differences}

(a) The basic conceptual idea behind $H_{\infty}$ design involves the minimisation of the magnitudes of a transfer function, which is quite different from the $H_{2} / L Q G$ requirement to minimise a complex domain integral representing error and control signal power spectra.

(b) The $H_{\infty}$ design approach is closer to that of classical frequency response design in that the frequency-response shaping of desired transfer functions is attempted.

(c) The calculation of $H_{\infty}$ controllers is more complicated than the equivalent $\mathrm{H}_{2} / L Q G$ controllers, whether this be in the time or frequency domains.

(d) Improvements in the robustness of $\mathrm{H}_{2} / L Q G$ designs to model inaccuracies must be achieved in a rather artificial manner. The $H_{\infty}$ approach is more suited to the design of controllers for systems with uncertainty. 


\subsubsection{Relationships between Classical Design and $H_{\infty}$ Robust Control}

There are many similarities between classical frequency domain design procedures [40] and $H_{\infty}$ robust design, which can be exploited, such as:

- Classical gain and phase margins can be related to sensitivity function magnitudes.

- The peak magnitude of the closed-loop frequency response $\left(H_{\infty}\right.$ norm of the complementary sensitivity) has often provided an indication of the likely overshoot on the step response.

- Robustness measures have been assessed in frequency domain terms with the distance from the critical (-1) point on a Nyquist diagram, providing a measure of robustness.

- Cost weightings in $H_{\infty}$ design can be parameterised to provide the same type of tuning knobs familiar in PID or lead/lag compensation.

- As in classical design, stochastic properties of the system must be shaped indirectly, using the target frequency response characteristics.

- Engineers trained in classical frequency domain design methods should be able to use $H_{\infty}$ design procedures easily (assuming formal design steps are followed).

\subsection{4 $\mathrm{H}_{2}$ and $\mathrm{H}_{\infty}$ Design and Relationship to PID Control}

A technique has been proposed [36], whereby the proportional, integral, derivative (PID) controller coefficients can be selected to improve $H_{\infty}$ robustness properties. For practical application of the $H_{\infty}$ technique the research results can often be translated into a simpler $P I D$ design algorithm. The advantage of such a procedure is that it offers the possibility of achieving more robust PID designs. A disadvantage is that a pure PID structure is not obtained, although in practice $P I D$ controllers are usually not implemented without additional filtering. The $P I D$ controller is valuable in many industrial applications, but robustness is not guaranteed. The use of $H_{\infty}$ techniques to enable the robustness of PID designs to be improved therefore has some merit. A more recent approach to designing $H_{2}$ and $H_{\infty}$ controllers, with a PID structure, is referred to as restricted structure optimal control [79]. In this approach a controller of restricted structure (RS) is optimised to minimise a given $H_{\infty}$ or $H_{2}$ cost index, which is very valuable for applications [80]. The same restricted structure ideas may be applied to feed-forward and tracking control problems [86]. Adaptive versions of such controllers are available [81], and the dual $R S$ estimation problems have also been considered [87, 88].

\subsection{5 $H_{\infty}$ Polynomial Systems Synthesis Theory}

The $H_{\infty}$ optimal control problems can be solved using a polynomial systems approach where the system is represented by a polynomial matrix. The philosophy was introduced by Kwakernaak [18, 43, 44] and the link between the linear quadratic gaussian (LQG) and $H_{\infty}$ problems was invoked by Grimble [31] to obtain a solution to the $H_{\infty}$ problem, using the relatively well-known $L Q G$ problem results. In fact, in one form the $H_{\infty}$ controller can be thought of as being an $L Q G$ controller, which is designed with special 
dynamic (transfer-function) cost weighting terms. The technique [43] originally employed for solving $H_{\infty}$ control problems is related to that introduced in Chapter 3. There is a difference in the philosophy adopted and the solution procedure, but the starting lemma and the resulting algorithms are essentially the same. Later, Kwakernaak [45] introduced a different strategy, which is rather similar to the use of the bounded real lemma in the statespace approach, described below. This employed J spectral factorisation and Kwakernaak [46] developed a software suite for calculating these controllers in polynomial matrix form. Many of these tools are now available in the PolyX Matlab toolbox.

\subsection{State-space Modelling and Synthesis Theory}

Most of this text is concerned with the polynomial systems approach to modelling and control design. However, the state-space methods are very popular and there are good reasons for using the most appropriate method for the particular application. In addition, state-space models may provide a natural representation for a new system design, where the physical system equations are readily available. Even in such a case the designer may of course choose to transform the system into a frequency domain form for the design process. The solution of $\mathrm{H}_{2}$ or $L Q$ optimal control problems, by state equation methods, is well known [82] and hence only the results for the $H_{\infty}$ design approach will be summarised here. The state-space results are useful for comparison purposes with the more complete presentation of the polynomial approach to $H_{\infty}$ control design later.

The monograph by Francis [22] provides a useful introduction to $H_{\infty}$ synthesis procedures, but the computational techniques described in the monograph have been superseded by the state-space-based Riccati equation approaches. The seminal paper on this latter method was by Doyle, Glover, Khargonekar and Francis [23]. Simple statespace formulas were derived for all controllers solving a standard continuous-time $H_{\infty}$ problem. That is, given a real number $\gamma$, all controllers such that the $H_{\infty}$ norm of a closed-loop transfer function is strictly less than $\gamma$ can be found by this approach. The smallest value of $\gamma$ must normally be obtained by iteration. The text by Stoorvogel [24] summarises the results for both discrete- and continuous-time systems.

It will now be helpful to introduce the basic state equation model and the discrete-time state-space solution, even though the following chapters are mainly concerned with the polynomial matrix representation of systems. This will enable the relationships between the two approaches to be investigated and a perspective on this important alternative method to be obtained [37]. The state-space results below are very important to the development of the subject of $H_{\infty}$ design. However, they are mainly included for completeness and theorems are quoted without proof and with limited explanation.

\subsubsection{State-space Solution of Discrete-time $H_{\infty}$ Control Problem}

The objective here is to provide a brief overview of the solution for the discrete multivariable $H_{\infty}$ control problem [25]. Consider the following discrete, time-invariant, plant:

$$
\begin{aligned}
x(t+1) & =\bar{A} x(t)+\bar{B}_{1} w(t)+\bar{B}_{2} u(t) \\
z(t) & =\bar{C}_{1} x(t)+\bar{D}_{12} u(t)
\end{aligned}
$$


where $t=0,1,2, \ldots$ and the state $x(t) \in R^{n}$, disturbance $w(t) \in R^{q}$, control $u(t) \in R^{m}$ and the generalised error (controlled output) $z(t) \in R^{p}$. For process control systems $u(t)$ would denote the manipulated valuable $(M V)$ and $z(t)$ would denote the process variable $(P V)$. The state equation matrices are given an overbar to distinguish them from the polynomial and transfer-function matrices, which are employed later.

Assumptions:

$$
\begin{aligned}
& \mathrm{AS}(\mathrm{i}) \quad\left(\bar{A}, \bar{B}_{2}\right) \text { is stabilisable, }\left(\bar{C}_{1}, \bar{A}\right) \text { detectable } \\
& \mathrm{AS}(\mathrm{ii}) \bar{D}_{12}^{T}\left[\bar{D}_{12} \bar{C}_{1}\right]=\left[\begin{array}{ll}
R_{1} & 0
\end{array}\right] \text { and } R_{1}>0 .
\end{aligned}
$$

The latter assumption assumes the cross-weighting between the control signal and the state vector is null.

\subsection{2 $H_{\infty}$ Control Design Objectives}

The aim is to find a control strategy $\{u(t)\}$ in $\ell_{2}[0, \infty)$ which leads to a bounded $H_{\infty}$ norm of the transfer function matrix $T_{z w}$ from input $w(t)$ to output $z(t)$. The control signal $u(t)=-C_{0}\left(z^{-1}\right) x(t)$, where $C_{0}\left(z^{-1}\right)$ represents the controller in negative feedback notation. Let the $z$-transfer to be minimised in a $H_{\infty}$ norm sense:

$$
T_{z w}=\left(\bar{C}_{1}-\bar{D}_{12} C_{0}\right)\left(z I-\bar{A}+\bar{B}_{2} C_{0}\right)^{-1} \bar{B}_{1}
$$

The aims of control may be summarised as:

(i) The constant-gain feedback controller $C_{0}$ should ensure the system is closed-loop stable and $\left(\bar{A}-\bar{B}_{2} C_{0}\right)$ is an asymptotically stable matrix.

(ii) The gain $T_{z w}$ must satisfy $\left\|T_{z w}\right\|_{\infty} \leq \gamma$ where $\gamma>0$.

The constraint on the $H_{\infty}$ norm will ensure, from the small-gain theorem [6], that the system will be robustly stable in a certain sense. That is, if $\Delta\left(z^{-1}\right)$ represents a stable transfer function matrix, satisfying the $H_{\infty}$ norm bound $\left\|\Delta\left(z^{-1}\right)\right\|_{\infty}<\gamma^{-1}$, then $\Delta\left(z^{-1}\right)$ may be connected from $z$ back to $w$ without destabilising the closed-loop system so formed. There are many results for different types of uncertainty structure that demonstrate the improvement which can be gained by minimising an $H_{\infty}$ norm. For example, Xie and de Souza [69] have discussed the control of linear state-space systems with norm-bounded time-varying uncertainty.

\subsubsection{State-feedback Control Solution}

The following lemma summarises the solution of the state feedback $H_{\infty}$ optimal control problem for systems represented in state equation form:

Lemma 1.1 $H_{\infty}$ state feedback control gain

Consider the state-feedback system described in Section 1.4.1 under the assumptions AS(i) and AS(ii). Suppose a matrix $P \in R^{n \times n}$ exists satisfying:

$$
\begin{aligned}
& P \geq 0 \quad \text { and } \quad \gamma^{2} I-\bar{B}_{1}^{T} P \bar{B}_{1}>0 \\
& P=\bar{A}^{T} P\left(I+\bar{B}_{2} R_{1}^{-1} \bar{B}_{2}^{T} P-\gamma^{-2} \bar{B}_{1} \bar{B}_{1}^{T} P\right)^{-1} \bar{A}+\bar{C}_{1}^{T} \bar{C}_{1}
\end{aligned}
$$


The state feedback $H_{\infty}$ optimal control: $u(t)=-K_{c 2} x(t)$ and the control gain:

$$
K_{c 2}=R_{1}^{-1} \bar{B}_{2}^{T} P\left(I+\bar{B}_{2} R_{1}^{-1} \bar{B}_{2}^{T} P-\gamma^{-2} \bar{B}_{1} \bar{B}_{1}^{T} P\right)^{-1} \bar{A}
$$

ensures $\left(\bar{A}-\bar{B}_{2} C_{0}\right)$ is asymptotically stable and $\left\|T_{z w}\right\|_{\infty} \leq \gamma$.

Proof Presented in Basar [26]. Note that the feedback controller in this case $C_{0}\left(z^{-1}\right)=K_{c 2}$.

The state-feedback $H_{\infty}$ control solution is illustrated in Figure 1.2. Note that the dotted loop represents the generation of a worst case disturbance signal. This loop is not part of the controller to be implemented, but arises in the game problem solution to the problem. This approach is discussed in more detail in Chapter 5. Without loss of generality it is assumed that $\gamma=1$ since $\bar{B}_{1}$ can always be rescaled by $\gamma^{-1}$ (letting $\bar{B}_{1 \gamma}=\gamma^{-1} \bar{B}_{1}$ ). An alternative and equally valuable form of the above results may be obtained by algebraic manipulation, by letting: $P=\left(I+\gamma^{-2} X \bar{B}_{1} \bar{B}_{1}^{T}\right)^{-1} X=X\left(I+\gamma^{-2} \bar{B}_{1} \bar{B}_{1}^{T} X\right)^{-1}$ where the matrix: $X \geq 0$. Then Equation (1.5) becomes:

$$
\begin{aligned}
P & =\bar{A}^{T} X\left(I+\gamma^{-2} \bar{B}_{1} \bar{B}_{1}^{T} X+\left(\bar{B}_{2} R_{1}^{-1} \bar{B}_{2}^{T}-\gamma^{2} \bar{B}_{1} \bar{B}_{1}^{T}\right) X\right)^{-1} \bar{A}+\bar{C}_{1}^{T} \bar{C}_{1} \\
& =\bar{A}^{T} X\left(I+\bar{B}_{2} R_{1}^{-1} \bar{B}_{2}^{T} X\right)^{-1} \bar{A}+\bar{C}_{1}^{T} \bar{C}_{1}
\end{aligned}
$$

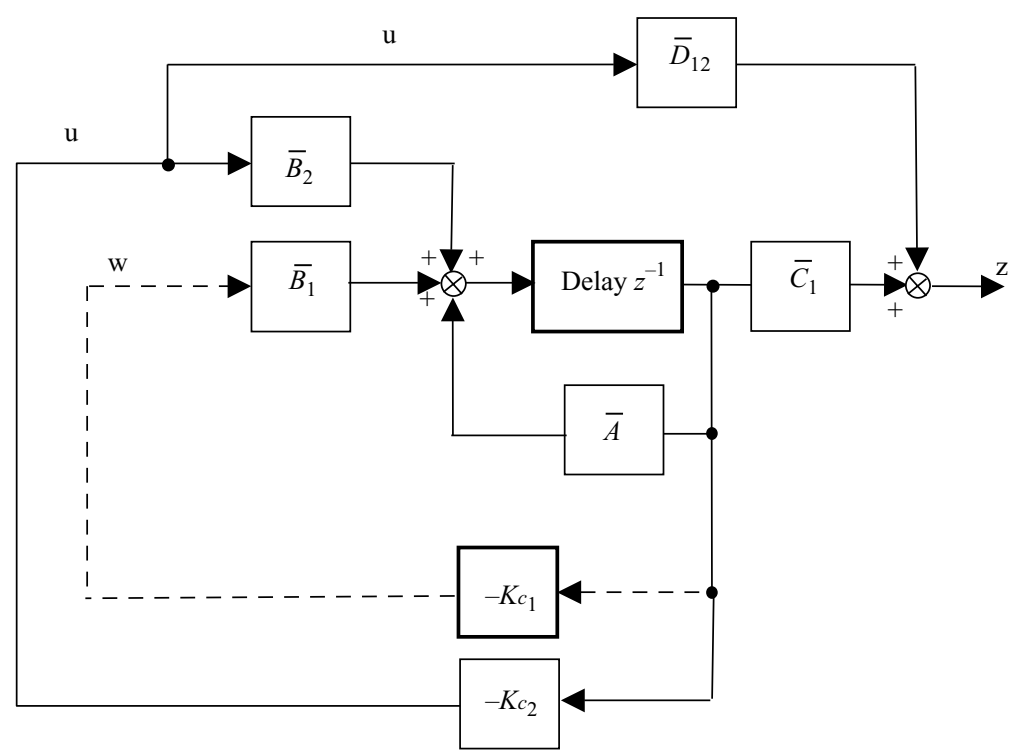

Figure 1.2 State-Feedback $H_{\infty}$ optimal control solution with fictitious worst case disturbance loop (dotted lines) 
but

$$
\left(I+\bar{B}_{2} R_{1}^{-1} \bar{B}_{2}^{T} X\right)^{-1}=I-\bar{B}_{2} R_{1}^{-1} \bar{B}_{2}^{T} X\left(I+\bar{B}_{2} R_{1}^{-1} \bar{B}_{2}^{T} X\right)^{-1}
$$

and

$$
\left(I+\gamma^{-2} \bar{B}_{1} \bar{B}_{1}^{T} X\right)^{-1}=I-\gamma^{-2} \bar{B}_{1} \bar{B}_{1}^{T} X\left(I-\gamma^{-2} \bar{B}_{1} \bar{B}_{1}^{T} X\right)^{-1}
$$

Hence,

$$
\begin{gathered}
X\left(I+\gamma^{-2} \bar{B}_{1} \bar{B}_{1}^{T} X\right)^{-1}=\bar{A}^{T} X\left(I+\bar{B}_{2} R_{1}^{-1} \bar{B}_{2}^{T} X\right)^{-1} \bar{A}+\bar{C}_{1}^{T} \bar{C}_{1} \\
=\bar{A}^{T} X\left(I-\bar{B}_{2} R_{1}^{-1} \bar{B}_{2}^{T} X\left(I+\bar{B}_{2} R_{1}^{-1} \bar{B}_{2}^{T} X\right)^{-1}\right) \bar{A}+\bar{C}_{1}^{T} \bar{C}_{1}
\end{gathered}
$$

or

$$
\begin{aligned}
& \bar{A}^{T} X \bar{A}-\bar{A}^{T} X \bar{B}_{2} R_{1}^{-1} \bar{B}_{2}^{T} X\left(I+\bar{B}_{2} R_{1}^{-1} \bar{B}_{2}^{T} X\right)^{-1} \bar{A} \\
& \quad+\bar{C}_{1}^{T} \bar{C}_{1}-X\left(I+\gamma^{-2} \bar{B}_{1} \bar{B}_{1}^{T} X\left(I+\gamma^{-2} \bar{B}_{1} \bar{B}_{1}^{T} X\right)^{-1}\right)=0
\end{aligned}
$$

The desired alternative form of the Riccati equation therefore becomes:

$$
\begin{aligned}
& \bar{A}^{T} X \bar{A}-X-\bar{A}^{T} X \bar{B}_{2}\left(I+R_{1}^{-1} \bar{B}_{2}^{T} X \bar{B}_{2}\right)^{-1} R_{1}^{-1} \bar{B}_{2}^{T} X \bar{A} \\
& +X \bar{B}_{1}\left(I+\gamma^{-2} \bar{B}_{1}^{T} X \bar{B}_{1}\right)^{-1} \gamma^{-2} \bar{B}_{1}^{T} X+\bar{C}_{1}^{T} \bar{C}_{1}=0
\end{aligned}
$$

and the controller gain expression:

$$
\begin{aligned}
K_{c 2} & =R_{1}^{-1} \bar{B}_{2} X\left(I+\gamma^{-2} \bar{B}_{1} \bar{B}_{1}^{T} X+\left(\bar{B}_{2} R_{1}^{-1} \bar{B}_{2}^{T}-\gamma^{-2} \bar{B}_{1} \bar{B}_{1}^{T}\right) X\right)^{-1} \bar{A} \\
& =R_{1}^{-1} \bar{B}_{2} X\left(I+\bar{B}_{2} R_{1}^{-1} \bar{B}_{2}^{T} X\right)^{-1} \bar{A}=\left(I+R_{1}^{-1} \bar{B}_{2}^{T} X \bar{B}_{2}\right)^{-1} R_{1}^{-1} \bar{B}_{2}^{T} X \bar{A}
\end{aligned}
$$

The following lemma may now be established, based on these results.

Lemma 1.2 $H_{\infty}$ state-feedback control gain calculation

Consider the system described in Section 1.4.1 and the assumptions AS(i) and AS(ii). Suppose a matrix $X \in R^{n \times n}$ exists satisfying: $X \geq 0$,

$$
\begin{aligned}
X= & \bar{A}^{T} X \bar{A}+\bar{C}_{1}^{T} \bar{C}_{1}-\bar{A}^{T} X \bar{B}_{2}\left(R_{1}+\bar{B}_{2}^{T} X \bar{B}_{2}\right)^{-1} \bar{B}_{2}^{T} X \bar{A} \\
& +X \bar{B}_{1}\left(\gamma^{2} I+\bar{B}_{1}^{T} X \bar{B}_{1}\right)^{-1} \bar{B}_{1}^{T} X
\end{aligned}
$$

The state-feedback $H_{\infty}$ optimal control $u(t)=-K_{c 2} x(t)$ and the state-feedback gain:

$$
K_{c 2}=\left(R_{1}+\bar{B}_{2}^{T} X \bar{B}_{2}\right)^{-1} \bar{B}_{2}^{T} X \bar{A}
$$

stabilises the closed-loop system and ensures $\left\|T_{z w}\right\|_{\infty} \leq \gamma$. 
Similarity between LQG and $\boldsymbol{H}_{\infty}$ synthesis problems: If the weightings: $Q_{1}=\bar{C}_{1}^{T} \bar{C}_{1}$ and $R_{1}=\bar{D}_{12}^{T} \bar{D}_{12}$ denote the error and control signal weightings in an $L Q G$ synthesis problem, the solution will be very similar to the state-feedback controller defined in Lemmas 1.1 and 1.2. In fact, when $\gamma \rightarrow \infty$ certain terms in the algebraic Riccati equations and gains tend to zero and the $H_{\infty}$ and $L Q$ controllers coincide. In later chapters a corresponding relationship is established between the $H_{\infty}$ and $L Q$ polynomial forms of these controllers.

\subsubsection{State-feedback Control Problem: Cross-product Costing Case}

If the system assumptions are modified, the equivalent of a cost function with crossproduct term between state and control signal can be introduced. That is, let the assumptions become:

Assumptions:

$\mathrm{AS}(\mathrm{iii}) \quad\left(\bar{A}, \bar{B}_{2}\right)$ is stabilisable, $\left(\bar{A}, \bar{C}_{1}\right)$ detectable

AS(iv) $\quad\left[\begin{array}{cc}\bar{A}-z I & \bar{B}_{2} \\ \bar{C}_{1} & \bar{D}_{12}\end{array}\right]$ has full column rank for all $z=e^{j \omega}$, where $\omega \in[0,2 \pi)$.

Let $\bar{D}_{12}^{T}\left[\begin{array}{ll}\bar{D}_{12} & \bar{C}_{1}\end{array}\right]=\left[\begin{array}{ll}R_{1} & G^{T}\end{array}\right]$ where $R_{1}>0$. The latter definition assumes the crossweighting matrix $G$, between the control signal and state, is not null. Also define the control and cross-product weightings $R_{c}$ and $G_{c}$ as:

$$
R_{c}=\left[\begin{array}{cc}
-\gamma^{2} I & 0 \\
0 & R_{1}
\end{array}\right] \quad \text { and } \quad G_{c}=\left[\begin{array}{ll}
0 & G
\end{array}\right]
$$

The main results for this case are summarised in the following Lemma.

Lemma 1.3 $H_{\infty}$ state-feedback control with cross-product costing Consider the state-feedback system described in Section 1.4.1 (Equations 1.1 and 1.2) under the assumptions: AS(iii) and AS(iv). Suppose a matrix $P \in R^{n \times n}$ exists, satisfying:

$$
\begin{aligned}
& \gamma^{2} I-\bar{B}_{1}^{T} P \bar{B}_{1}>0, \quad P \geq 0 \\
& P=\bar{A}^{T} P \bar{A}+\bar{C}_{1}^{T} \bar{C}_{1}-\left(\bar{A}^{T} P \bar{B}+G_{c}\right)\left(R_{c}+\bar{B}^{T} P \bar{B}\right)^{-1}\left(\bar{B}^{T} P \bar{A}+G_{c}^{T}\right)
\end{aligned}
$$

The state-feedback $H_{\infty}$ control signal $u(t)=-K_{c 2} x(t)$ and the control gain:

$$
K_{c}=\left[\begin{array}{l}
K_{c 1} \\
K_{c 2}
\end{array}\right]=\left(R_{c}+\bar{B}^{T} P \bar{B}\right)^{-1}\left(\bar{B}^{T} P \bar{A}+G_{c}^{T}\right)
$$

where $K_{c 1} \in R^{q \times n}$ and $K_{c 2} \in R^{m \times n}$, and gain ensures: $\bar{A}-\bar{B}_{2} K_{c 2}$ is asymptotically stable and $\left\|T_{z w}\right\|_{\infty} \leq \gamma$.

Proof Presented in Basar [26] and Mustafa and Bernstein [27]. 
This lemma reduces to Lemma 1.1 when the cross-product term $G$ is set to zero. The solution of the discrete-time $H_{\infty}$ control problem was also considered by Iglesias and Glover [28].

\subsubsection{State-space Solution of Discrete-time $H_{\infty}$ Filtering Problem}

Attention may now turn to the dual optimal filtering problems. If an observations signal $\{y(t)\}$ is available, representing a plant output corrupted by measurement noise, the plant equations become:

$$
\begin{aligned}
& x(t+1)=\bar{A} x(t)+\bar{B}_{1} w(t)+\bar{B}_{2} u(t) \\
& y(t)=\bar{C}_{2} x(t)+\bar{D}_{21} w(t)
\end{aligned}
$$

where $t=0,1,2, \ldots$ and the state $x(t) \in R^{n}$, disturbance $w(t) \in R^{q}$, control $u(t) \in R^{m}$ and observations $y(t) \in R^{r}$. The so-called exogenous input signal $\{w(t)\}$ represents an unknown square summable sequence. The $H_{\infty}$ filtering problem is to obtain an estimate for the signal: $z(t)=\bar{C}_{1} x(t)+\bar{D}_{12} u(t)$ that is optimal in the sense that the transfer function matrix $T_{\varepsilon w}$, from $w(t)$ to $\varepsilon(t)$, defined by:

$$
\varepsilon(t)=z(t)-\hat{z}(t)
$$

is bounded by $\left\|T_{z w}\right\|_{\infty} \leqslant \gamma$, where only the values of $\{y(\tau)\}, \tau<t$, can be used for the estimation of $\{z(t)\}$.

Assumptions:

$\mathrm{AS}(\mathrm{v}) \quad\left(\bar{A}, \bar{B}_{1}\right)$ is stabilisable, $\left(\bar{A}, \bar{C}_{2}\right)$ detectable

$\mathrm{AS}(\mathrm{vi}) \quad\left[\begin{array}{cc}\bar{A}-z I & \bar{B}_{1} \\ \bar{C}_{2} & \bar{D}_{21}\end{array}\right]$ has full column rank for all $z=e^{j \omega}$, where $\omega \in[0,2 \pi)$.

Let $\left[\begin{array}{c}\bar{B}_{1} \\ \bar{D}_{21}\end{array}\right] \bar{D}_{21}^{T}=\left[\begin{array}{c}S \\ R_{2}\end{array}\right]$ where $R_{2}>0$. Notice that the cross-covariance term may not be null in this problem which is the dual of that considered in the previous section. Also define the weightings $R_{f}$ and $S_{f}$ as:

$$
R_{f}=\left[\begin{array}{cc}
-\gamma^{2} I & 0 \\
0 & R_{2}
\end{array}\right] \text { and } S_{f}=\left[\begin{array}{ll}
0 & S
\end{array}\right]
$$

$\boldsymbol{H}_{\infty}$ Filter: the state-space form of the $H_{\infty}$ filter has a structure that is the same as for a model-based estimator in observer form, and this is the same as a Kalman filter, but with the gain chosen in a particular way. The estimator equations are presented below:

$$
\begin{aligned}
\hat{x}(t+1) & =\bar{A} \hat{x}(t)+K_{f 2}\left(y(t)-\bar{C}_{2} \hat{x}(t)\right)+\bar{B}_{2} u(t) \\
\hat{z}(t) & \left.=\bar{C}_{1} \hat{x}(t)\right)+\bar{D}_{12} u(t) \\
\varepsilon(t) & =z(t)-\hat{z}(t)=\bar{C}_{1}[x(t)-\hat{x}(t)]
\end{aligned}
$$


From Equations (1.1) and (1.17), the state estimation error becomes:

$$
\begin{aligned}
\tilde{x}(t+1) & =x(t+1)-\hat{x}(t+1) \\
& =\bar{A} \tilde{x}(t)+\bar{B}_{1} w(t)-K_{f 2}\left[y(t)-\bar{C}_{2} \hat{x}(t)\right] \\
& =\bar{A} \tilde{x}(t)+\bar{B}_{1} w(t)-K_{f 2}\left[\bar{C}_{2} \tilde{x}(t)+\bar{D}_{21} w(t)\right]
\end{aligned}
$$

or

$$
\tilde{x}(t)=\left(z I-\bar{A}+K_{f 2} \bar{C}_{2}\right)^{-1}\left(\bar{B}_{1}-K_{f 2} \bar{D}_{21}\right) w(t)
$$

but from Equation (1.19): $\varepsilon(t)=\bar{C}_{1} \tilde{x}(t)$. It follows that the transfer function matrix $T_{\varepsilon w}$ to be minimised in an $H_{\infty}$ sense is given as:

$$
T_{\varepsilon w}=\bar{C}_{1}\left(z I-\bar{A}+K_{f 2} \bar{C}_{2}\right)^{-1}\left(\bar{B}_{1}-K_{f 2} \bar{D}_{21}\right)
$$

The dual result to the control Lemma 1.2 may now be given, which is for the case where the cross-product term $(S)$ is null.

\section{Lemma $1.4 H_{\infty}$ filtering state-space problem Solution}

Consider the system (1.1), (1.2) and filter (1.17), (1.18), under the assumptions: AS(v) and AS(vi). Suppose a matrix $X \in R^{n \times n}$ exists satisfying: $X \geq 0$

$$
X=\bar{A} X \bar{A}^{T}+\bar{B}_{1} \bar{B}_{1}^{T}-\bar{A} X \bar{C}_{2}^{T}\left(R_{2}+\bar{C}_{2} X \bar{C}_{2}^{T}\right) \bar{C}_{2} X \bar{A}^{T}+X \bar{C}_{1}\left(\gamma^{2} I+\bar{C}_{1} X \bar{C}_{1}^{T}\right)^{-1} \bar{C}_{1} X
$$

The $H_{\infty}$ filter gain can be computed as:

$$
K_{f 2}=\bar{A} X \bar{C}_{2}^{T}\left(R_{2}+\bar{C}_{2} X \bar{C}_{2}^{T}\right)^{-1}
$$

where $K_{f 2} \in R^{n \times r}$ and the gain ensures: $\left(\bar{A}-K_{f 2} \bar{C}_{2}\right)$ is asymptotically stable and $\left\|T_{\varepsilon w}\right\|_{\infty} \leq \gamma$.

Without loss of generality, it may be assumed that $\gamma=1$, since $\bar{C}_{1}$ and $\bar{D}_{12}$ can always be rescaled by $\gamma^{-1}$, replacing $\bar{C}_{1}$ and $\bar{D}_{12}$ by $\gamma^{-1} \bar{C}_{1}$ and $\gamma^{-1} \bar{D}_{12}$, respectively. The more general form of the filter which includes the cross-weighting term is presented below. This is the dual of the $H_{\infty}$ control problem given in Lemma 1.3.

Lemma 1.5 $H_{\infty}$ filtering state-space problem solution

Consider the system (1.1), (1.2) and the filter (1.17), (1.18), under the assumptions: AS(v) and $\mathrm{AS}(\mathrm{vi})$. Suppose a matrix $Z \in R^{n \times n}$ exists satisfying:

$$
\begin{aligned}
& \gamma^{2} I-\bar{C}_{1} Z \bar{C}_{1}^{T}>0 \\
& Z=\bar{A} Z \bar{A}^{T}+\bar{B}_{1} \bar{B}_{1}^{T}-\left(\bar{A} Z \bar{C}^{T}+S_{f}\right)\left(R_{f}+\bar{C} Z \bar{C}^{T}\right)^{-1}\left(\bar{C} Z \bar{A}^{T}+S_{f}^{T}\right)
\end{aligned}
$$


Then the filter gain $K_{f 2}$ can be found from the following partition:

$$
K=\left[\begin{array}{ll}
K_{f 1} & K_{f 2}
\end{array}\right]=\left(\bar{A} Z \bar{C}^{T}+S_{f}\right)\left(R_{f}+\bar{C} Z \bar{C}^{T}\right)^{-1}
$$

where $K_{f 1} \in R^{n \times p}, K_{f 2} \in R^{n \times r}$ and gain ensures: $\left(\bar{A}-K_{f 2} \bar{C}_{2}\right)$ is asymptotically stable and $\left\|T_{\varepsilon w}\right\|_{\infty} \leq \gamma$.

The $H_{\infty}$ filter enables an $H_{\infty}$ bound on the estimation error to be guaranteed. Comparison of these results with those of the usual discrete-time Kalman filtering problem [29] reveals that the equations are almost identical. The main difference lies in the definition of $R_{f}$, which includes the scalar $\gamma^{2}$ for the $H_{\infty}$ case. It may easily be confirmed that as $\gamma^{2} \rightarrow \infty$ the filter tends to a Kalman filter for this system. A similar $H_{\infty}$ filter may be defined which uses the current measurement of $y(t)$ to compute the estimate of $x(t)$. The solution of the $H_{\infty}$ filtering problem is illustrated in Figure 1.3. The dotted loop represents the generation of a worst case observations signal and innovations signal, which arises in the game theory solution of the problem, discussed in Chapter 8.

\subsubsection{Bounded Real Lemma}

When solving the linear quadratic ( $L Q)$ optimal control problems, the Riccati equation can be introduced in a completing the squares minimisation argument. Despite the simplicity of the state-space $H_{\infty}$ Riccati equation results, the conceptual link between the $H_{\infty}$ problem and the Riccati equation solution is not so easy to justify physically, as is the case in the $L Q G$ problem case. The previous results were obtained from a game theory approach, but a different perspective on the problem can be found using the bounded real lemma. Petersen, Anderson and Jonckheere [30] used the bounded real lemma to provide a direct link between the $H_{\infty}$ problem and the Riccati equations in a first principles solution. The following Lemmas are due to Yaesh and Shaked [25]. The assumptions made by these authors were the same as AS(i) and AS(ii), with the exception that controllability and observability replace the stabilisable and detectable assumptions, respectively.

Lemma 1.6 Bounded Real Lemma

Let the transfer function matrix be:

$$
G\left(z^{-1}\right)=\bar{C}(z I-\bar{A})^{-1} \bar{B}
$$

where $\bar{A}$ is a nonsingular stability matrix and $(\bar{A}, \bar{B}, \bar{C})$ is observable. Then, $\left\|G\left(z^{-1}\right)\right\|_{\infty}<\gamma$ if and only if there exists a positive definite solution $\Sigma$ to the following quadratic matrix equation:

$$
\Sigma=\bar{A}^{T} \Sigma \bar{A}+\Sigma \bar{B}\left(\gamma^{2} I+\bar{B}^{T} \Sigma \bar{B}\right)^{-1} \bar{B}^{T} \Sigma+\bar{C}^{T} \bar{C}
$$




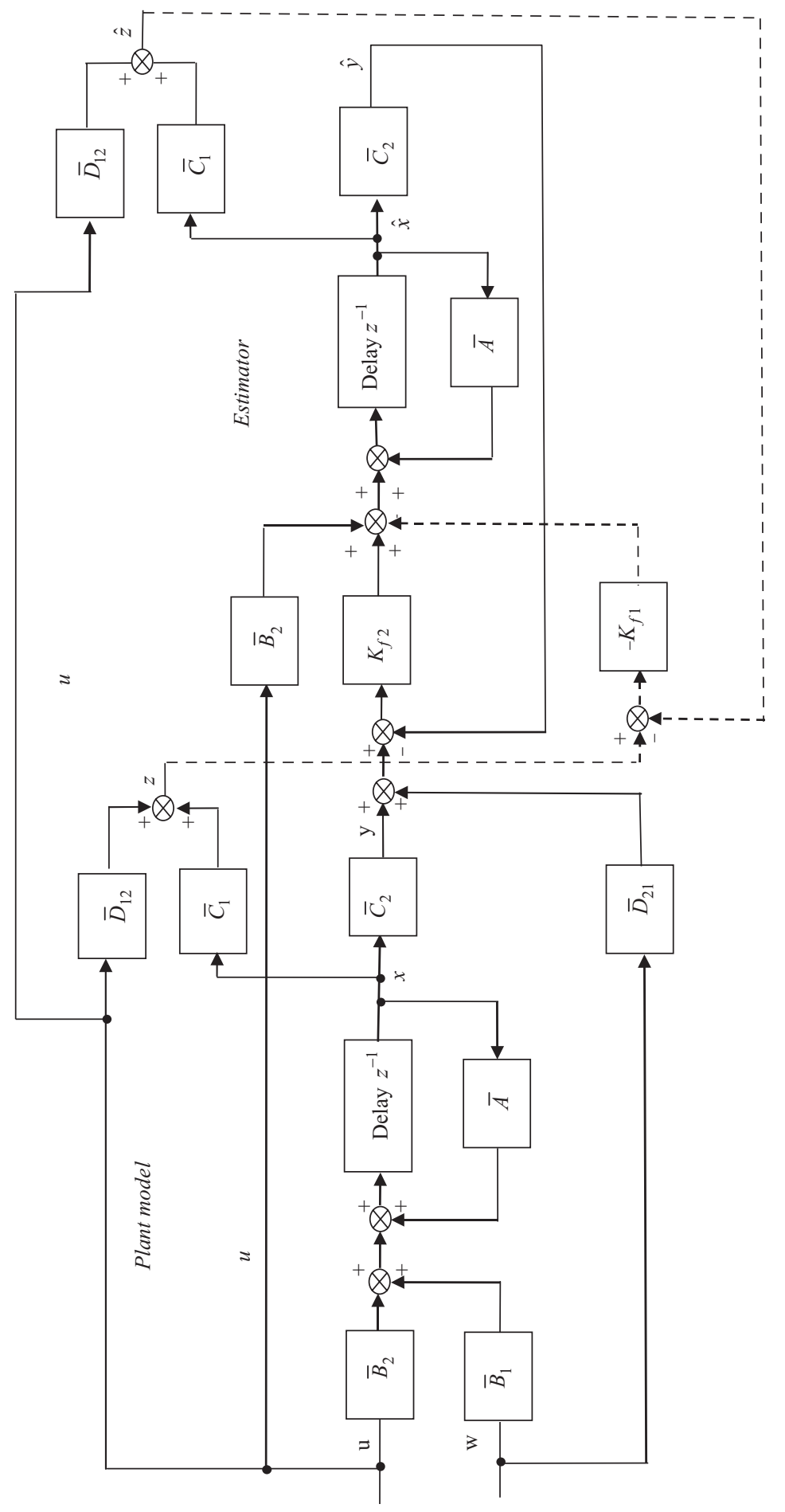

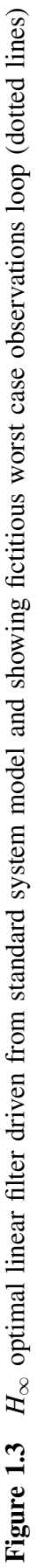


It may readily be shown that the feedback control, which ensures the $H_{\infty}$ norm is less than $\gamma$, is a constant matrix $C_{0}=K_{c 2}$. Thus, the bounded real lemma [38] may be invoked by identifying terms in Equations (1.3) and (1.27). That is, let $\bar{C} \rightarrow\left(\bar{C}_{1}-\bar{D}_{12} K_{c 2}\right), \bar{A} \rightarrow \bar{A}-\bar{B}_{2} K_{c 2}$ and $\bar{B} \rightarrow \bar{B}_{1}$. Note from Lemma 1.6 that $\left\|T_{z w}\right\|_{\infty} \leq \gamma$ if and only if there exists a $\Sigma>0$, that satisfies:

$$
\Sigma=\left(\bar{A}-\bar{B}_{2} K_{c 2}\right)^{T} \Sigma\left(\bar{A}-\bar{B}_{2} K_{c 2}\right)+\Sigma \bar{B}_{1}\left(\gamma^{2} I+\bar{B}_{1}^{T} \Sigma \bar{B}_{1}\right)^{-1} \bar{B}_{1}^{T} \Sigma+\bar{C}_{1}^{T} \bar{C}_{1}+K_{c 2}^{T} R_{1} K_{c 2}
$$

Following a completing the squares argument obtain:

$$
\Sigma=\bar{A}^{T} \Sigma \bar{A}+\Sigma \bar{B}_{1}\left(\gamma^{2} I+\bar{B}_{1}^{T} \Sigma \bar{B}_{1}\right)^{-1} \bar{B}_{1}^{T} \Sigma-\bar{A}^{T} \Sigma \bar{B}_{2}\left(R_{1}+\bar{B}_{2}^{T} \Sigma \bar{B}_{2}\right)^{-1} \bar{B}_{2}^{T} \Sigma \bar{A}+\bar{C}_{1}^{T} \bar{C}_{1}+S_{0}
$$

where

$$
S_{0}=\left[K_{c 2}^{T}-\bar{A}^{T} \Sigma \bar{B}_{2}\left(R_{1}+\bar{B}_{2}^{T} \Sigma \bar{B}_{2}\right)^{-1}\right]\left(R_{1}+\bar{B}_{2}^{T} \Sigma \bar{B}_{2}\right)\left[K_{c 2}-\left(R_{1}+\bar{B}_{2}^{T} \Sigma \bar{B}_{2}\right)^{-1} \bar{B}_{2}^{T} \Sigma \bar{A}\right]
$$

It may then easily be shown that a stabilising solution, which satisfies $\left\|T_{z w}\right\|_{\infty} \leq \gamma$ exists, if and only if, there exists $\Sigma>0$ that satisfies the algebraic Riccati equation:

$$
\Sigma=\bar{A}^{T} \Sigma \bar{A}+\Sigma \bar{B}_{1}\left(\gamma^{2} I+\bar{B}_{1}^{T} \Sigma \bar{B}_{1}\right)^{-1} \bar{B}_{1}^{T} \Sigma-\bar{A}^{T} \Sigma \bar{B}_{2}\left(R_{1}+\bar{B}_{2}^{T} \Sigma \bar{B}_{2}\right)^{-1} \bar{B}_{2}^{T} \Sigma \bar{A}+\bar{C}_{1}^{T} \bar{C}_{1}
$$

If there exists a positive definite solution $\Sigma$ to Equation (1.31) then the norm result is guaranteed by the stabilising gain $K_{c 2}$ which sets $S_{0}$ to zero, namely:

$$
K_{c 2}=\left(R_{1}+\bar{B}_{2}^{T} \Sigma \bar{B}_{2}\right)^{-1} \bar{B}_{2}^{T} \Sigma \bar{A}
$$

The results here are clearly comparable with those given earlier in Lemma 1.2, obtained from a game theory approach. These are different merits of the game theory and the bounded real lemma approaches. The game problem solution also provides a link to $\mathrm{H}_{2}$ optimisation, whilst the bounded real lemma gives a very direct solution, as the above analysis suggests. A dual version of Lemma 1.6 was also given by Yaesh and Shaked [25].

Lemma 1.7 Bounded real lemma (dual form)

Let $G\left(z^{-1}\right)$ be given by (1.27), and $\left(\bar{A}^{T}, \bar{C}^{T}, \bar{B}^{T}\right)$ satisfy the assumptions of Lemma 1.6. Then $\left\|G\left(z^{-1}\right)\right\|_{\infty}<\gamma$ if and only if there exists a positive definite solution $\Sigma$ to the following quadratic matrix equation:

$$
\Sigma=\bar{A} \Sigma \bar{A}^{T}+\Sigma \bar{C}^{T}\left(\gamma^{2} I+\bar{C} \Sigma \bar{C}^{T}\right)^{-1} \bar{C} \Sigma+\overline{B B}^{T}
$$


The above lemma may be used to obtain the solution of the $H_{\infty}$ filtering problem, by noting from Equation (1.21): $\bar{C} \rightarrow \bar{C}_{1}, \bar{A} \rightarrow \bar{A}-K_{f 2} \bar{C}_{2}$ and $\bar{B} \rightarrow\left(\bar{B}_{1}-K_{f 2} \bar{D}_{21}\right)$. Substituting in the quadratic Equation (1.33) and completing squares in the manner described for the control problem, the desired Riccati equation solution $\Sigma$ and gain $K_{f 2}$ can be found as:

$$
\Sigma=\bar{A} \Sigma \bar{A}^{T}+\bar{B}_{1} \bar{B}_{1}^{T}-\bar{A} \Sigma \bar{C}_{2}^{T}\left(R_{2}+\bar{C}_{2} \Sigma \bar{C}_{2}^{T}\right)^{-1} \bar{C}_{2} \Sigma \bar{A}^{T}+\Sigma \bar{C}_{1}^{T}\left(\gamma^{2} I+\bar{C}_{1} \Sigma \bar{C}_{1}^{T}\right)^{-1} \bar{C}_{1} \Sigma
$$

where $K_{f 2}=A \Sigma \bar{C}_{2}^{T}\left(R_{2}+\bar{C}_{2} \Sigma \bar{C}_{2}^{T}\right)^{-1}$. These results therefore provide a bounded real lemma approach to proving Lemma 1.4 .

\subsubsection{Output Feedback $H_{\infty}$ Control Problem}

The general $H_{\infty}$ control problem set-up which includes the output feedback problem can now be introduced. Consider a standard system model transfer-function matrix $G\left(z^{-1}\right)$, which maps exogenous inputs $w$ and control input $u$ to controlled outputs $z$ and measured outputs $y$. That is,

$$
\left[\begin{array}{l}
z \\
y
\end{array}\right]=G\left(z^{-1}\right)\left[\begin{array}{l}
w \\
u
\end{array}\right]
$$

where the transfer-function matrix:

$$
G\left(z^{-1}\right)=\left[\begin{array}{ll}
G_{11}\left(z^{-1}\right) & G_{12}\left(z^{-1}\right) \\
G_{21}\left(z^{-1}\right) & G_{22}\left(z^{-1}\right)
\end{array}\right]=\left[\begin{array}{ll}
\bar{D}_{11} & \bar{D}_{12} \\
\bar{D}_{21} & \bar{D}_{22}
\end{array}\right]+\left[\begin{array}{l}
\bar{C}_{1} \\
\bar{C}_{2}
\end{array}\right](z I-\bar{A})^{-1}\left[\begin{array}{ll}
\bar{B}_{1} & \bar{B}_{2}
\end{array}\right]
$$

and $\bar{A} \in R^{n \times n}$ and $z, y, w$ and $u$ are vectors of size $r_{1}, r_{2}$ and $m_{1}, m_{2}$, respectively. The assumption is normally made that $m_{1} \geq r_{2}$ and $r_{1} \geq m_{2}$. That is, there are more disturbances than measured outputs and more outputs being controlled than control signals. These assumptions are equivalent to assuming that full rank noise and weighting models are employed.

When the closed-loop output feedback control law $u=-C_{0}\left(z^{-1}\right) y$ is added to the standard system model $G\left(z^{-1}\right)$, the resulting linear fractional transformation (LFT), relating the input $w$ to the output $z$, becomes:

$$
T_{z w}\left(z^{-1}\right)=G_{11}-G_{12} C_{0}\left(I+G_{22} C_{0}\right)^{-1} G_{21}
$$

The sub-optimal $H_{\infty}$ control problem with parameter $\gamma$ therefore consists of finding a dynamic real-rational controller $C_{0}\left(z^{-1}\right)$, such that the feedback control law $u=-C_{0} y$ internally stabilises $G\left(z^{-1}\right)$ and makes the closed loop system gain less than $\gamma\left(\left\|T_{\mathrm{zw}}\left(z^{-1}\right)\right\|_{\infty} \leq \gamma\right)$. The solvability of this problem for $\gamma$ large enough only requires $\left(\bar{A}, \bar{B}_{2}, \bar{C}_{2}\right)$ be stabilisable and detectable. However, to avoid technical difficulties it is 
helpful to make the common assumptions: $\mathrm{AS}(\mathrm{iii})-\mathrm{AS}(\mathrm{vi})$ and to let $\bar{D}_{11}=0$ and $\bar{D}_{22}=0$. The additional assumption that the system $\left(\bar{A}, \bar{B}_{1}, \bar{C}_{1}\right)$ is minimal can also simplify analysis, but is less common. Assume that a minimal realisation of the controller $C_{0}\left(z^{-1}\right)$ is to be employed of the form:

$$
C_{0}\left(z^{-1}\right)=\bar{D}_{c}+\bar{C}_{c}\left(z I-\bar{A}_{c}\right)^{-1} \bar{B}_{c}
$$

Then a realisation of $T_{z w}$ follows as:

$$
T_{z w}\left(z^{-1}\right)=\bar{D}_{c 1}+\bar{C}_{c 1}\left(z I-\bar{A}_{c 1}\right)^{-1} \bar{B}_{c 1}
$$

where, under the above assumptions $\left(\bar{D}_{11}=0, \bar{D}_{22}=0\right)$ :

$$
\begin{array}{ll}
\bar{A}_{c 1}=\left[\begin{array}{cc}
\bar{A}-\bar{B}_{2} \bar{D}_{2} \bar{C}_{2} & -\bar{B}_{c} \bar{C}_{2} \\
\bar{B}_{c} \bar{C}_{2} & \bar{A}_{c}
\end{array}\right] \quad \bar{B}_{c 1}=\left[\begin{array}{c}
\bar{B}_{1}-\bar{B}_{2} \bar{D}_{c} \bar{D}_{21} \\
\bar{B}_{c} \bar{D}_{21}
\end{array}\right] \\
\bar{C}_{c 1}=\left[\begin{array}{ccc}
\bar{C}_{1}-\bar{D}_{12} \bar{D}_{c} & \bar{C}_{2}-\bar{D}_{12} \bar{C}_{c}
\end{array}\right] & \bar{D}_{c 1}=-\bar{D}_{12} \bar{D}_{c} \bar{D}_{21}
\end{array}
$$

Strategy for output feedback problem solution: the solution of the output feedback problem involves two stages:

(a) Define two new signals $\left\{r_{1}(t)\right\}$ and $\left\{u_{1}(t)\right\}$, where the $H_{\infty}$ norm: $\left\|T_{z w}\right\|_{\infty}=\left\|T_{v_{1} r_{1}}\right\|_{\infty}$.

(b) Show that $\left\|T_{v_{1} r_{1}}\right\|_{\infty}$ can be minimised by solving the $H_{\infty}$ filtering problem whose solution has already been given.

In the following section the signals $\left\{r_{1}(t)\right\}$ and $\left\{v_{1}(t)\right\}$ are defined. The system defined by Equations (1.1), (1.2) and (1.14) will again be considered.

Transfer function with equivalent $\boldsymbol{H}_{\infty}$ norm: consider the term $\delta V(t)=x^{T}(t+1)$ $P x(t+1)-x^{T}(t) P x(t)$, where $P=P^{T} \geq 0$ satisfies conditions (1.10) and (1.11) of Lemma 1.3. Thus, adding and subtracting terms:

$$
\delta V(t)=x^{T}(t+1) P x(t+1)-x^{T}(t) P x(t)+\|z(t)\|^{2}-\|z(t)\|^{2}+\gamma^{2}\left(\|w(t)\|^{2}-\|w(t)\|^{2}\right)
$$

but from Equation (1.2): $\|z(t)\|^{2}=x^{T}(t) \bar{C}_{1}^{T} \bar{C}_{1} x(t)+u^{T}(t) \bar{D}_{12}^{T} \bar{D}_{12} u(t)+2 u^{T}(t) \bar{D}_{12}^{T} \bar{C}_{1} x(t)$. Substituting in (1.41), using this and (1.1), obtain:

$$
\begin{aligned}
\delta V(t)= & -\|z(t)\|^{2}+\gamma^{2}\|w(t)\|^{2}+x^{T}(t)\left[\bar{A}^{T} P \bar{A}-P+\bar{C}_{1}^{T} \bar{C}_{1}\right] x(t) \\
& +u^{T}(t)\left[\bar{D}_{12}^{T} \bar{D}_{12}+\bar{B}_{2}^{T} P \bar{B}_{2}\right] u(t)-w^{T}(t)\left[\gamma^{2} I-\bar{B}_{1}^{T} P \bar{B}_{1}\right] w(t) \\
& +2 w^{T}(t) \bar{B}_{1}^{T} P \bar{B}_{2} u(t)+2 w^{T}(t) \bar{B}_{1}^{T} P \bar{A} x(t)+2 u^{T}(t)\left[\bar{D}_{12}^{T} \bar{C}_{1}+\bar{B}_{2}^{T} P \bar{A}\right] x(t)
\end{aligned}
$$


Letting $\tilde{R}=\left(R_{c}+\bar{B}^{T} P \bar{B}\right)$, then from Equations (1.11) and (1.12): $\bar{A}^{T} P \bar{A}-P$ $+\bar{C}_{1}^{T} \bar{C}_{1}=K_{c}^{T} \tilde{R} K_{c}$ and substituting into Equation (1.42):

$$
\begin{aligned}
& \delta V(t)+\|z(t)\|^{2}-\gamma^{2}\|w(t)\|^{2}=x^{T}(t) K_{c}^{T} \tilde{R} K_{c} x(t)+2\left[w^{T}(t) \quad u^{T}(t)\right] \\
& \times\left(\left[\begin{array}{c}
\bar{B}_{1}^{T} \\
\bar{B}_{2}^{T}
\end{array}\right] P \bar{A}+\left[\begin{array}{c}
0 \\
\bar{D}_{12}^{T} \bar{C}_{1}
\end{array}\right]\right) x(t) \\
& +\left[\begin{array}{ll}
w^{T}(t) & u^{T}(t)
\end{array}\right]\left(\left[\begin{array}{l}
\bar{B}_{1}^{T} \\
\bar{B}_{2}^{T}
\end{array}\right] P\left[\begin{array}{cc}
\bar{B}_{1} & \bar{B}_{2}
\end{array}\right]+\left[\begin{array}{cc}
-\gamma^{2} I & 0 \\
0 & R_{1}
\end{array}\right]\right)\left[\begin{array}{l}
w(t) \\
u(t)
\end{array}\right] \\
& =x^{T}(t) K_{c}^{T} \tilde{R} K_{c} x(t)+2\left[w^{T}(t) \quad u^{T}(t)\right] \tilde{R} K_{c} x(t)+2\left[w^{T}(t) \quad u^{T}(t)\right] \tilde{R}\left[\begin{array}{l}
w(t) \\
u(t)
\end{array}\right] \\
& =\left(x^{T}(t) K_{c}^{T}+\left[\begin{array}{ll}
w^{T}(t) & u^{T}(t)
\end{array}\right]\right) \tilde{R}\left(K_{c} x(t)+\left[\begin{array}{l}
w(t) \\
u(t)
\end{array}\right]\right)
\end{aligned}
$$

To simplify this expression introduce the signals $\left\{r_{2}(t)\right\}$ and $\left\{v_{2}(t)\right\}$ as: $r_{2}(t)=w(t)+$ $K_{c 1} x(t)$ and $v_{2}(t)=u(t)+K_{c 2} x(t)$. Then, recalling (1.12), Equation (1.43) becomes:

$$
\begin{aligned}
& \delta V(t)+\|z(t)\|^{2}-\gamma^{2}\|w(t)\|^{2}=\left[\begin{array}{ll}
r_{2}^{T}(t) & v_{2}^{T}(t)
\end{array}\right] \tilde{R}\left[\begin{array}{l}
r_{2}(t) \\
v_{2}(t)
\end{array}\right] \\
& =v_{2}^{T}(t)\left(R_{1}+\bar{B}_{2}^{T} P \bar{B}_{2}+\bar{B}_{2}^{T} P \bar{B}_{1} \theta_{1}^{-1} \bar{B}_{1}^{T} P \bar{B}_{2}\right) v_{2}(t) \\
& -\left(r_{2}^{T}(t) \theta_{1}^{1 / 2}-v_{2}^{T}(t) \bar{B}_{2}^{T} P \bar{B}_{1} \theta_{1}^{-1 / 2}\right)\left(\theta_{1}^{1 / 2} r_{2}(t)-\theta_{1}^{-1 / 2} \bar{B}_{1}^{T} P \bar{B}_{2} v_{2}(t)\right)
\end{aligned}
$$

where the following variables are defined as:

$$
\begin{aligned}
& \theta_{1}=\gamma^{2} I-\bar{B}_{1}^{T} P \bar{B}_{1} \\
& \theta_{2}=R_{1}+\bar{B}_{2}^{T}\left(P+P \bar{B}_{1} \theta_{1}^{-1} \bar{B}_{1}^{T} P\right) \bar{B}_{2}
\end{aligned}
$$

The signals of interest then become:

$$
\begin{aligned}
v_{1}(t) & =\theta_{2}^{1 / 2} v_{2}(t)=\theta_{2}^{1 / 2}\left(u(t)+K_{c 2} x(t)\right) \\
r_{1}(t) & =\theta_{1}^{1 / 2} r_{2}(t)-\theta_{1}^{-1 / 2} \bar{B}_{1}^{T} P \bar{B}_{2} v_{2}(t) \\
& =\theta_{1}^{1 / 2} w(t)-\theta_{1}^{-1}\left(\bar{B}_{1}^{T} P \bar{B}_{2} u(t)-\left[\theta_{1} K_{c 1}-\bar{B}_{1}^{T} P \bar{B}_{2} K_{c 2}\right] x(t)\right)
\end{aligned}
$$

Note that the last term in this equation may be written as:

$$
\left[-\theta_{1} K_{c 1}+\bar{B}_{1}^{T} P \bar{B}_{2} K_{c 2}\right]=\left[\left(-\gamma^{2} I+\bar{B}_{1}^{T} P \bar{B}_{1}\right) \bar{B}_{1}^{T} P \bar{B}_{2}\right] K_{c}
$$

but $\tilde{R} \tilde{R}^{-1}=I$ and hence:

$$
\left[\left(-\gamma^{2} I+\bar{B}_{1}^{T} P \bar{B}_{1}\right) \bar{B}_{1}^{T} P \bar{B}_{2}\right] \tilde{R}^{-1}=\left[\begin{array}{ll}
I & 0
\end{array}\right]
$$

so that

$$
\left[\begin{array}{ll}
I & 0
\end{array}\right]\left(\bar{B}^{T} P \bar{A}+G_{c}^{T}\right)=\bar{B}_{1}^{T} P \bar{A}
$$


Thus, from (1.49) and (1.50):

$$
\left[-\theta_{1} K_{c 1}+\bar{B}_{1}^{T} P \bar{B}_{2} K_{c 2}\right]=\bar{B}_{1}^{T} P \bar{A}
$$

and $r_{1}(t)$ follows from (1.48):

$$
r_{1}(t)=\gamma^{-1} \theta_{1}^{1 / 2}\left(w(t)-\theta_{1}^{-1} \bar{B}_{1}^{T} P\left(\bar{B}_{2} u(t)+\bar{A} x(t)\right)\right)
$$

Equation (1.44) may now be written in terms of (1.47) and (1.48) as:

$$
\delta V(t)+\|z(t)\|^{2}-\gamma^{2}\|w(t)\|^{2}=\left\|v_{1}(t)\right\|^{2}-\gamma^{2}\left\|r_{1}(t)\right\|^{2}
$$

Summing both sides of the last equality from $t=0, \ldots, \infty$ with $x(0)=x(\infty)=0$ gives:

$$
\|z(t)\|_{2}^{2}-\gamma^{2}\|w(t)\|_{2}^{2}=\left\|v_{1}(t)\right\|_{2}^{2}-\gamma^{2}\left\|r_{1}(t)\right\|_{2}^{2}
$$

This result, together with the definition of the $H_{\infty}$ norm in the Appendix reveals that the $H_{\infty}$ norm of the transfer $T_{z w}\left(z^{-1}\right)$ is the same as the $H_{\infty}$ norm of $T_{v_{1} r_{1}}\left(z^{-1}\right)$.

Equivalent system model: Equations (1.1), (1.2) and (1.14) may now be written in an equivalent form by noting:

$$
w(t)=\gamma \theta_{1}^{-1 / 2} r_{1}(t)+\theta_{1}^{-1} \bar{B}_{1}^{T} P\left(\bar{B}_{2} u(t)+\bar{A} x(t)\right) \quad \text { and } \quad u(t)=\theta_{2}^{-1 / 2} v_{1}(t)-K_{c 2} x(t)
$$

hence

$$
\begin{aligned}
x(t+1) & =\left(I+\bar{B}_{1} \theta_{1}^{-1} \bar{B}_{1}^{T} P\right) \bar{A} x(t)+\bar{B}_{1} \gamma \theta_{1}^{-1 / 2} r_{1}(t)+\left(I+\bar{B}_{1} \theta_{1}^{-1} \bar{B}_{1}^{T} P\right) \bar{B}_{2} u(t) \\
v_{1}(t) & =\theta_{2}^{1 / 2}\left(K_{c 2} x(t)+u(t)\right) \\
y(t) & =\left(\bar{C}_{2}+\bar{D}_{21} \theta_{1}^{-1} \bar{B}_{1}^{T} P \bar{A}\right) x(t)+\bar{D}_{21} \gamma \theta_{1}^{-1 / 2} r_{1}(t)+\bar{D}_{21} \theta_{1}^{-1} \bar{B}_{1}^{T} P \bar{B}_{2} u(t)
\end{aligned}
$$

Define the new system state equation matrices as: $\tilde{A}=\left(I+\bar{B}_{1} \theta_{1}^{-1} \bar{B}_{1}^{T} P\right) \bar{A}, \tilde{B}_{1}=$ $\bar{B}_{1} \gamma \theta_{1}^{-1 / 2}$ and $\tilde{B}_{2}=\left(I+\bar{B}_{1} \theta_{1}^{-1} \bar{B}_{1}^{T} P\right) \bar{B}_{2}, \quad \tilde{C}_{1}=\theta_{2}^{1 / 2} K_{c 2}$ and $\tilde{C}_{2}=\bar{C}_{2}+\bar{D}_{21} \theta_{1}^{-1} \bar{B}_{1}^{T} P \bar{A}$, $\tilde{D}_{12}=\theta_{2}^{1 / 2}, \tilde{D}_{21}=\bar{D}_{21} \gamma \theta_{1}^{-1 / 2}, \tilde{D}_{22}=\bar{D}_{21} \theta_{1}^{-1} \bar{B}_{1}^{T} P \bar{B}_{2}$. The system may then be expressed in the equivalent state equation form:

$$
\begin{aligned}
x(t+1) & =\tilde{A} x(t)+\tilde{B}_{1} r_{1}(t)+\tilde{B}_{2} u(t) \\
v_{1}(t) & =\tilde{C}_{1} x(t)+\tilde{D}_{12} u(t) \\
y(t) & =\tilde{C}_{2} x(t)+\tilde{D}_{21} r_{1}(t)+\tilde{D}_{22} u(t)
\end{aligned}
$$

Observer-based compensator: the following observed-based compensator may now be introduced for the system presented in the previous section:

$$
\begin{aligned}
\hat{x}(t+1) & =\tilde{A} \hat{x}(t)+\tilde{B}_{2} u(t)+K_{f 2}\left(y(t)-\tilde{C}_{2} \hat{x}(t)-\tilde{D}_{22} u(t)\right) \\
u(t) & =-K_{c 2} \hat{x}(t)
\end{aligned}
$$


Define the state estimation error $\tilde{x}(t)=x(t)-\hat{x}(t)$, then from (1.56) and (1.59), the evolution of the state is given by:

$$
\begin{aligned}
\tilde{x}(t+1) & =\tilde{A} \tilde{x}(t)+\tilde{B}_{1} r_{1}(t)-K_{f 2}\left(\tilde{C}_{2} \tilde{x}(t)+\tilde{D}_{21} r_{1}(t)\right) \\
& =\left(\tilde{A}-K_{f 2} \tilde{C}\right) \tilde{x}(t)+\left(\tilde{B}_{1}-K_{f 2} \tilde{D}_{21}\right) r_{1}(t)
\end{aligned}
$$

and from (1.57) and (1.60):

$$
v_{1}(t)=\tilde{C}_{1} x(t)-\tilde{D}_{12} K_{c 2} \hat{x}(t)=\tilde{C}_{1} \tilde{x}(t)
$$

The observer-based compensator results in a transfer-function matrix from $r_{1}$ to $v_{1}$ given by:

$$
T_{v_{1} r_{1}}\left(z^{-1}\right)=\tilde{C}_{1}\left(z I-\tilde{A}+K_{f 2} \tilde{C}_{2}\right)^{-1}\left(\tilde{B}_{1}-K_{f 2} \tilde{D}_{21}\right)
$$

The solution of the output feedback control problem may now be given by invoking the $H_{\infty}$ filtering results of Lemma 1.5. Note first that the presence of the matrix $\tilde{D}_{22}$ in Equation (1.58) leads to the introduction of the same term in the estimation Equation (1.59), but otherwise $\tilde{D}_{22}$ does not affect the $H_{\infty}$ filtering equations discussed previously.

Lemma 1.8 $H_{\infty}$ output feedback control law

Consider the system described by the state Equations (1.1), (1.2) and (1.14) under the assumptions AS(iii) and AS(iv). If there exists $P \geq 0$ and $K_{c 2}$ satisfying the conditions of Lemma 1.3 and $Z \geq 0$ satisfying:

$$
\begin{aligned}
& \gamma^{2} I-\tilde{C}_{1} Z \tilde{C}_{1}^{T}>0 \\
& Z=\tilde{A} Z \tilde{A}^{T}+\tilde{B}_{1} \tilde{B}_{1}^{T}-\left(\tilde{A} Z \tilde{C}^{T}+\tilde{S}_{f}\right)\left(\tilde{R}_{f}+\tilde{C} Z \tilde{C}^{T}\right)^{-1}\left(\tilde{C} Z \tilde{A}^{T}+\tilde{S}_{f}^{T}\right)
\end{aligned}
$$

where

$$
\left[\begin{array}{c}
\tilde{S} \\
\tilde{R}_{2}
\end{array}\right]=\left[\begin{array}{c}
\tilde{B}_{1} \\
\tilde{D}_{21}
\end{array}\right] \tilde{D}_{21}^{T}, \tilde{R}_{f}=\left[\begin{array}{cc}
-\gamma^{2} I & 0 \\
0 & \tilde{R}_{2}
\end{array}\right], \quad \tilde{S}_{f}=\left[\begin{array}{ll}
0 & \tilde{S}
\end{array}\right]
$$

and if the system matrices are defined earlier as in Section 1.4.7, then the compensator:

$$
\begin{aligned}
\hat{x}(t+1) & =\bar{A}_{c} \hat{x}(t)+\bar{B}_{c} y(t) \\
u(t) & =\bar{C}_{c} \hat{x}(t)
\end{aligned}
$$

where the triple $\left(\bar{A}_{c}, \bar{B}_{c}, \bar{C}_{c}\right)$ :

$$
\begin{aligned}
& \bar{A}_{c}=\tilde{A}-K_{f 2} \tilde{C}_{2}-\left(\tilde{B}_{2}-K_{f 2} \tilde{D}_{22}\right) K_{c 2} \\
& \bar{B}_{c}=K_{f 2} \\
& \bar{C}_{c}=-K_{c 2}
\end{aligned}
$$

stabilises the closed-loop system, such that: $\left\|T_{z w}\left(z^{-1}\right)\right\|_{\infty} \leq \gamma$

Proof The above results may be collected to prove the $H_{\infty}$ norm bound is satisfied. 
Stability of the closed-loop system can be proved by using the straightforward argument in Furuta and Phoojaruenchanachai [38]. Yaesh and Shaked [68] have considered the output feedback problem using a state-space discrete-time approach.

\subsection{Introduction to $\mathrm{H}_{2}$ or $L Q G$ Polynomial Synthesis}

In the next chapter a solution is presented to the key theoretical problem, namely the linear quadratic gaussian ( $L Q G)$ design problem, for a very general system and cost function. These results can be specialised to many particular industrial design problems for one, two, two-and-a-half degrees-of-freedom controllers and for minimum variance, $L Q G$ and generalised $L Q G$ cost functions. General theoretical results are valuable since they can provide the solutions of a range of important special cases. They are also useful for developing the software which can solve a range of problems. However, for pedagogical reasons a simple solution of the $L Q G$ problem will now be considered for a simpler problem description. This is obtained, leaving aside many of the technical issues, to enable the solution technique to be obtained easily. Note that it is now common to refer to the general solution of linear quadratic optimal control problems as $\mathrm{H}_{2}$ optimisation and the more traditional linear quadratic deterministic or stochastic control problems as being $L Q R$ or $L Q G$ control, respectively. In the following, $H_{2}$ or $L Q G$ design are used almost interchangeably.

\subsubsection{System Description}

The single-input single-output (SISO) stochastic system to be controlled will now be introduced. Both tracking and regulating problems are considered and the system is represented by a mixed autoregressive moving average process, with the single-degreeof-freedom (DOF) structure shown in Figure 1.4. The reference, disturbance and measurement noise signals are assumed to be stable and are denoted by $r, d$ and $v$, respectively. The noise-free output $y$ is to be controlled using the cascade controller $C_{0}=C_{0 d}^{-1} C_{0 n}$. The plant $z$-transfer function is denoted:

$$
W\left(z^{-1}\right)=A\left(z^{-1}\right)^{-1} B\left(z^{-1}\right)
$$

where $z$ is the $z$-transform complex number $z=e^{s T_{s}}$ and $T_{s}$ is the sample time. The plant can be unstable, nonminimum phase and may include a time-delay of

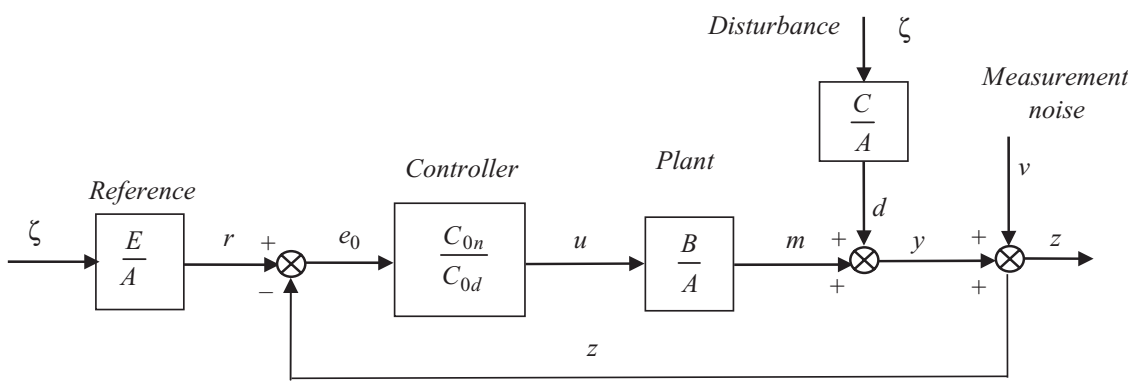

Figure 1.4 Discrete-time closed-loop unity feedback control system 
magnitude $k \geq 1$. The plant numerator and denominator polynomials are assumed to be of the form:

$$
\begin{aligned}
& B\left(z^{-1}\right)=z^{-k}\left(b_{0}+b_{1} z^{-1}+\cdots+b_{n_{b}-k} z^{-\left(n_{b}-k\right)}\right), \quad\left(b_{n_{b}-k} \neq 0\right) \\
& A\left(z^{-1}\right)=1+a_{1} z^{-1}+a_{2} z^{-2}+\cdots+a_{n_{a}} z^{-n_{a}}
\end{aligned}
$$

The orders of the plant polynomials are assumed to be known and $n_{a}, n_{b} \geq 1$.

The disturbance and reference subsystems are denoted by $W_{d}\left(z^{-1}\right)=A\left(z^{-1}\right)^{-1} C\left(z^{-1}\right)$ and $W_{r}\left(z^{-1}\right)=A\left(z^{-1}\right)^{-1} E\left(z^{-1}\right)$, respectively, where the arguments of the polynomials are omitted for notational simplicity. The polynomial $A\left(z^{-1}\right)$ represents the least common denominator for all the above subsystems and by assumption none of these subsystems may include unstable hidden modes. The signals $v(t), \xi(t)$ and $\zeta(t)$ are assumed to be stationary, zero-mean sequences of uncorrelated random variables which are jointly independent and have the variances $R, Q_{2}$ and $Q_{3}$, respectively.

For the sake of brevity the arguments of time functions and of operators are often suppressed and as noted earlier, the indeterminate $z^{-1}$ is used to denote the $z$-transform complex number, or the unit-delay operator in the time-domain and which is appropriate should be clear from the context of its use. Further explanation of the notation and the fundamental mathematical results required is given in the Appendix.

So-called generalised spectral factors (Appendix, Sections A1.3 and A1.4) are required in the following analysis and are defined below. First note that the performance criterion error and control weighting functions are constants and denoted by $Q_{c}$ and $R_{c}$, respectively. The generalised spectral factors are defined as minimum-phase transfer functions, which satisfy:

$$
\begin{aligned}
& Y_{f} Y_{f}^{*}=\left(E Q_{3} E^{*}+C Q_{2} C^{*}+A R A^{*}\right) /\left(A A^{*}\right) \\
& Y_{c}^{*} Y_{c}=\left(B^{*} Q_{c} B+A^{*} R_{c} A\right) /\left(A^{*} A\right)
\end{aligned}
$$

where if $Y_{f}=Y_{f}\left(z^{-1}\right)$ then in the scalar case $Y_{f}^{*}=Y_{f}(z)$. The polynomials $D_{f}$ and $D_{c}$ are $S c h u r$, or so-called stable, polynomials, $\left(1 / D_{f}\right.$ and $1 / D_{c}$ are stable transfer functions) and satisfy:

$$
\begin{aligned}
& Y_{f} Y_{f}^{*}=D_{f} D_{f}^{*} /\left(A A^{*}\right) \Rightarrow Y_{f}=D_{f} / A \\
& Y_{c}^{*} Y_{c}=D_{c}^{*} D_{c} /\left(A^{*} A\right) \Rightarrow Y_{c}=D_{c} / A
\end{aligned}
$$

The role the spectral factors play should become clear in the following completing-thesquares solution.

System equations: the controller input is denoted by:

$$
e_{0}=r-z
$$

and the tracking error:

$$
e=r-y
$$


The plant equations can now be expressed, using the unity-variance white noise signal $\{\varepsilon(t)\}$, in a form which will be termed the innovations signal model:

$$
A e_{0}=D_{f} \varepsilon-B u
$$

\subsubsection{Cost Function and Solution}

The $H_{2} / L Q G$ cost function and optimal controller are defined first [82] and this is followed by the optimal solution. The controller is chosen to minimise the steady-state $\left(t_{0} \rightarrow-\infty\right)$ cost function:

$$
J=E\left\{Q_{c} e^{2}(t)+R_{c} u^{2}(t)\right\}
$$

where $E\{\cdot\}$ denotes the unconditional expectation operator. The constant weighting scalars: $Q_{c}, R_{c}>0$.

Theorem 1.1 LQG Scalar Optimal Controller

Consider the performance criterion (Equation 1.82) and the system described in Section 1.5.1, which excluded the presence of unstable hidden modes and which is in the steady-state condition. The optimal controller is given as:

$$
C_{0}=H_{0}^{-1} G_{0}
$$

The following coupled Diophantine equations, in terms of the unknown polynomials, $G, H$ and $F$, provide the unique particular solution $G_{0}, H_{0}$, with $F_{0}$ of smallest degree:

$$
\begin{aligned}
& D_{c}^{*} z^{-g} G+F A=B^{*} Q_{c} D_{f} z^{-g} \\
& D_{c}^{*} z^{-g} H-F B=A^{*} R_{c} D_{f} z^{-g}
\end{aligned}
$$

where $g=\max \left(n_{d_{c}}, n_{b}, n_{a}\right)$. These equations can be combined to obtain the implied equation:

$$
A H+B G=D_{c} D_{f}
$$

and the closed-loop system characteristic polynomial: $\rho_{c}=D_{c} D_{f}$.

Proof The proof which follows is in two stages: the minimisation of the performance criterion and the solution of the Diophantine equations. Recall that Diophantine equations and polynomial systems theory are reviewed in the Appendix.

\subsubsection{Minimisation of the Performance Criterion}

A brief review of the solution for the $H_{2} / L Q G$ controller, using the polynomial approach, is given below. The controller input, or error signal, is denoted by $e_{0}=r-z$ and the 
control sensitivity function $M$ is defined as:

$$
M=C_{0} /\left(1+C_{0} W\right)
$$

Thus,

$$
1-W M=1 /\left(1+C_{0} W\right)
$$

The control, plant output and tracking error signals become:

$$
\begin{aligned}
& u=M(r-v-d) \\
& y=(1-W M) d+W M(r-v) \\
& e=(1-W M)(r-d)+W M v
\end{aligned}
$$

Cost function expansion: under steady-state conditions the cost expression (Equation 1.82) can be written, using a discrete form of Parseval's theorem, as:

$$
E\left\{Q_{c} e^{2}(t)+R_{c} u^{2}(t)\right\}=\frac{1}{2 \pi j} \oint_{|z|=1}\left\{Q_{c} \Phi_{e e}\left(z^{-1}\right)+R_{c} \Phi_{u u}\left(z^{-1}\right)\right\} \frac{\mathrm{d} z}{z}
$$

where the contour integral is taken around the unit-circle $(|z|=1)$ in the $z$-plane, and $\Phi_{e e}\left(z^{-1}\right)$ and $\Phi_{u u}\left(z^{-1}\right)$ denote the power spectral densities of the signals $\{e(t)\}$ and $\{u(t)\}$, respectively. Substituting from Equations (1.89) and (1.91) and neglecting the uncorrelated noise terms, note that,

$$
\Phi_{e e}=(1-W M)\left(\Phi_{r r}+\Phi_{d d}\right)(1-W M)^{*}+W M \Phi_{v v}(W M)^{*}
$$

and

$$
\Phi_{u u}=M\left(\Phi_{r r}+\Phi_{v v}+\Phi_{d d}\right) M^{*}
$$

Substituting the spectral terms in the expression for the cost function, and simplifying, obtain:

$$
\begin{aligned}
E\left\{Q_{c} e^{2}(t)+R_{c} u^{2}(t)\right\}= & \frac{1}{2 \pi j} \oint_{|z|=1}\left\{Q _ { c } \left[(1-W M)\left(\Phi_{r r}+\Phi_{d d}\right)\left(1-W^{*} M^{*}\right)\right.\right. \\
& \left.\left.+W M \Phi_{v v} W^{*} M^{*}\right]+R_{c} M\left(\Phi_{r r}+\Phi_{v v}+\Phi_{d d}\right) M^{*}\right\} \frac{\mathrm{d} z}{z} \\
= & \frac{1}{2 \pi j} \oint_{|z|=1}\left\{Y_{c}^{*} Y_{c} M M^{*} Y_{f} Y_{f}^{*}+Q_{c}\left(1-W M-W^{*} M^{*}\right) \Phi_{0}\right\} \frac{\mathrm{d} z}{z}
\end{aligned}
$$

where $\Phi_{0}=\Phi_{r r}+\Phi_{d d}$. This expression was simplified by substituting for the spectral factors $Y_{c}$ and $Y_{f}$ (from Equations 1.77 and 1.78). This enables the completing-the- 
squares step so that the criterion can be written as:

$$
\begin{aligned}
J= & \frac{1}{2 \pi j} \oint_{|z|=1}\left\{\left(Y_{c} M Y_{f}-\frac{Q_{c} \Phi_{0} W^{*}}{Y_{c}^{*} Y_{f}^{*}}\right)\left(Y_{c} M Y_{f}-\frac{Q_{c} \Phi_{0} W^{*}}{Y_{c}^{*} Y_{f}^{*}}\right)^{*}\right. \\
& \left.+Q_{c} \Phi_{0}\left(1-\frac{Q_{c} \Phi_{0} W W^{*}}{Y_{c} Y_{c}^{*} Y_{f} Y_{f}^{*}}\right)\right\} \frac{\mathrm{d} z}{z}
\end{aligned}
$$

The completion-of-squares argument used above is straightforward and can easily be checked by multiplying out both of the expressions to show they are identical. Note that the final two terms are independent of $M$ and do not enter into the minimisation procedure. The term $Q_{c} \Phi_{0} W^{*} /\left(Y_{c}^{*} Y_{f}^{*}\right)$ may now be expanded by substituting for the transfers as:

$$
Q_{c} \Phi_{0} W^{*} /\left(Y_{c}^{*} Y_{f}^{*}\right)=Q_{c}\left(\frac{D_{f} B^{*}}{A D_{c}^{*}}-\frac{R A^{*} B^{*}}{D_{c}^{*} D_{f}^{*}}\right)
$$

The second term in Equation (1.97) is unstable, but if the plant is open-loop unstable the first term includes both stable and unstable components. For the optimisation procedure the first term in Equation (1.97) must be split up (using a type of partialfraction expansion procedure) into stable and unstable terms. This requires the introduction of a polynomial equation (in the indeterminate $z^{-1}$ ), which enables this decomposition to occur. The next equation introduced satisfies this requirement. In fact the opportunity will be taken to introduce the three polynomial equations, which are involved in the optimality and stability arguments.

Diophantine equations: assume that the following Diophantine equations are satisfied:

$$
\begin{aligned}
& D_{c}^{*} z^{-g} G+F A=B^{*} Q_{c} D_{f} z^{-g} \\
& D_{c}^{*} z^{-g} H-F B=A^{*} R_{c} D_{f} z^{-g}
\end{aligned}
$$

where the polynomials $\{H, G, F\}$ represent the solution, with $F$ of smallest degree, and $g=\max \left\{n_{d_{c}}, n_{b}, n_{a}\right\}$. From adding the above equations and employing (1.76) obtain the implied Diophantine equation: $A H+B G=D_{c} D_{f}$. Equation (1.97) may now be simplified, using (1.98), to obtain: $Q_{c} \Phi_{0} W^{*} /\left(Y_{c}^{*} Y_{f}^{*}\right)=G / A+\left(F z^{g} / D_{c}^{*}-Q_{c} R A^{*} B^{*} /\left(D_{c}^{*} D_{f}^{*}\right)\right)$. From this and (1.97) and writing the controller: $C_{0}=C_{0 d}^{-1} C_{0 n}$ obtain:

$$
\left(Y_{c} M Y_{f}-\frac{Q_{c} \Phi_{0} W^{*}}{Y_{c}^{*} Y_{f}^{*}}\right)=\frac{D_{c} D_{f} C_{0 n}}{A\left(A C_{0 d}+B C_{0 n}\right)}-\frac{G}{A}-\left(\frac{F z^{g}}{D_{c}^{*}}-\frac{Q_{c} R A^{*} B^{*}}{D_{c}^{*} D_{f}^{*}}\right)
$$

Equation (1.100) may be simplified, using (1.86), to obtain:

$$
\left(Y_{c} M Y_{f}-\frac{Q_{c} \Phi_{0} W^{*}}{Y_{c}^{*} Y_{f}^{*}}\right)=\frac{\left[C_{0 n} H-C_{0 d} G\right]}{A C_{0 d}+B C_{0 n}}-\left(\frac{F z^{g}}{D_{c}^{*}}-\frac{Q_{c} R A^{*} B^{*}}{D_{c}^{*} D_{f}^{*}}\right)
$$


Optimisation argument: the closed-loop system is assumed to be asymptotically stable and thence the characteristic polynomial $\left(A C_{0 d}+B C_{0 n}\right)$ is strictly stable. The first term on the right of (1.101) is therefore stable and is denoted by $T_{1}=[\cdot]$. The final term includes unstable transfers and is denoted by $T_{2}=(\cdot)$. The latter term is required to be analytic within the unit-circle for the optimisation procedure, but since $F$ is a minimal degree solution, $n_{f}<g$ and this condition is satisfied. The cost function may now be written as:

$$
J=\frac{1}{2 \pi j} \oint_{|z|=1}\left\{T_{1} T_{1}^{*}+T_{2} T_{2}^{*}-T_{2} T_{1}^{*}-T_{1} T_{2}^{*}+Q_{c} \Phi_{0}\left(1-\frac{Q_{c} \Phi_{0} W W^{*}}{Y_{c} Y_{c}^{*} Y_{f} Y_{f}^{*}}\right)\right\} \frac{\mathrm{d} z}{z}
$$

and by assumption $J<\infty$. The integral around the unit-circle of the term $T_{1} T_{2}^{*}$, which is analytic outside the unit-circle, may be found by the residue theorem [64] to be zero. Similarly the integral of the term $T_{2} T_{1}^{*}$ is null. The only term in the cost function which depends upon the controller is therefore the first term. It follows that the cost function is minimised when

$$
T_{1}=0 \quad \text { or } \quad C_{0}=C_{0 d}^{-1} C_{0 n}=H_{0}^{-1} G_{0}
$$

The minimum value of the cost function then becomes:

$$
J_{\min }=\frac{1}{2 \pi j} \oint_{|z|=1}\left\{T_{2} T_{2}^{*}+Q_{c} \Phi_{0}-Q_{c}^{2} \Phi_{0}^{2} W W^{*} /\left(Y_{c} Y_{c}^{*} Y_{f} Y_{f}^{*}\right)\right\} \frac{\mathrm{d} z}{z}
$$

\subsubsection{Solution of the Diophantine Equations and Stability}

The two coupled Diophantine equations introduced earlier have a unique solution [17] and this may now be investigated. Let the equations be written as:

$$
\left[\begin{array}{ccc}
z^{-g} D_{c}^{*} & A & 0 \\
0 & -B & z^{-g} D_{c}^{*}
\end{array}\right]\left[\begin{array}{c}
G \\
F \\
H
\end{array}\right]=\left[\begin{array}{l}
B^{*} z^{-g} Q_{c} D_{f} \\
A^{*} z^{-g} R_{c} D_{f}
\end{array}\right]
$$

It is well known that the system of linear equations: $T X=N$ has a solution if and only if the $\operatorname{rank} T=\operatorname{rank}[T, N]$. To check this condition it is necessary to look at all the values of $z$ for which $T$ and $[T, N]$ may have different ranks. Now the greatest common divisors $(G C D s)$ of all $1 \times 1$ and $2 \times 2$ minors are 1 and $D_{c}^{*} z^{-g}$, respectively, for both $T$ and $[T, N]$. Clearly the rank of both matrices is at least unity. The only value of $\mathrm{z}$ for which $T$ is rank 1 is a $G C D$ of $T$, which is this same as $G C D$ of $[T, N]$. Thence the rank of $T$ is the same as the rank of $[\mathrm{T}, \mathrm{N}]$. It follows that the above equations have a solution.

To show that the solution for $G, F$, and $H$ is unique note that $\left[-A, z^{-g} D_{c}^{*}, B\right]^{T}$ is a basis for the kernel of $T$. Let $G_{0}, F_{0}$ and $H_{0}$ denote the particular solution, so that

$$
\left[\begin{array}{c}
G \\
F \\
H
\end{array}\right]=\left[\begin{array}{c}
G_{0} \\
F_{0} \\
H_{0}
\end{array}\right]+\left[\begin{array}{c}
-A \\
z^{-g} D_{c}^{*} \\
B
\end{array}\right] p\left(z^{-1}\right)
$$


where $p\left(z^{-1}\right)$ is an arbitrary polynomial. Now the particular solution $G_{0}, F_{0}$ and $H_{0}$ satisfying $n_{f}<g$ is unique, since $F_{0}$ is the least degree representative of $F$ modulo $z^{-g} D_{c}^{*}$. Note that in the following analysis the existence of a unique solution to the Diophantine equations is not discussed, since the above steps may easily be repeated for the particular problem. The characteristic polynomial determining stability of the closed-loop system becomes:

$$
A C_{0 d}+B C_{0 n}=A H_{0}+B G_{0}=D_{c} D_{f}
$$

and it follows that the system is asymptotically stable and that $G_{0}$ and $H_{0}$ have no common unstable hidden modes. The error and control signals are also asymptotically stable, since:

$$
e=\left(1-\frac{B G_{0}}{D_{c} D_{f}}\right)(r-d)+\frac{B G_{0}}{D_{c} D_{f}} v \quad \text { and } \quad u=\frac{G_{0} A}{D_{c} D_{f}}(r-d-v)
$$

Continuous-Time System LQG Equations: if the system is continuous-time the optimisation procedure is almost identical to that presented previously. The only additional requirement is to prove the terms being minimised are strictly proper. The equations which result are very similar to those in Theorem 1.1. Simply replacing $z^{-1}$ with $s$ and defining $g=0$ (so that $z^{-g}=1$ ) provides the desired solution. This confirms the assertion that these results could be derived for the discrete-time case, in the knowledge that the results for the continuous-time problem would follow immediately. A similar situation will apply to all of the results which follow.

\subsection{5 $\mathrm{H}_{2} / L Q G$ Design Examples}

Simple examples follow for the design and computation of $H_{2} / L G Q$ optimal control laws.

Example 1.5.1 $L Q G$ optimal controller numerical calculation This example is based upon a nonminimum phase system represented in discrete ARMAX form:

$$
\left(1+a_{1} z^{-1}\right) y(t)=z^{-k}\left(1+b_{1} z^{-1}\right) u(t)+\left(1+c_{1} z^{-1}\right) \xi(t)
$$

where $z^{-1}$ represents the unit-delay operator and $a_{1}=-0.95, b_{1}=2, c_{1}=-0.7$ and $k=2$. The performance criterion weighting polynomials are defined as: $Q_{c}=1$ and $R_{c}=$ $\rho>0$. The white noise signals have covariances that are defined as: $Q_{2}=1$ and $R=\mu>0(r=0)$.

Solution: the spectral factors may first be derived from (1.75)-(1.78) to obtain:

$$
\begin{aligned}
D_{f} D_{f}^{*} & =\left(1+c_{1} z^{-1}\right) Q_{2}\left(1+c_{1} z\right)+\mu\left(1+a_{1} z^{-1}\right)\left(1+a_{1} z\right) \\
& =\left(Q_{2} c_{1}+\mu a_{1}\right)\left(z^{-1}+m+z\right)
\end{aligned}
$$

where $m=\left[Q_{2}\left(1+c_{1}^{2}\right)+\mu\left(1+a_{1}^{2}\right)\right] /\left(Q_{2} c_{1}+\mu a_{1}\right)$. Thence,

$$
D_{f} D_{f}^{*}=\sigma^{2}\left(\alpha+z^{-1}\right)(\alpha+z)
$$


where $\sigma^{2}=\left(Q_{2} c_{1}+\mu a_{1}\right) / \alpha$. Let $\alpha$ denote the solution $(\alpha>1)$ of the equation: $\alpha=\left[m \pm\left(m^{2}-4\right)^{1 / 2}\right] / 2$, then $D_{f}\left(z^{-1}\right)=\sigma\left(\alpha+z^{-1}\right), \sigma>0$. Similarly, obtain the control spectral factor as:

$$
\begin{aligned}
D_{c} D_{c}^{*} & =\left(1+b_{1} z\right) Q_{c}\left(1+b_{1} z^{-1}\right)+\rho\left(1+a_{1} z\right)\left(1+a_{1} z^{-1}\right) \\
& =\left(Q_{c} b_{1}+\rho a_{1}\right)\left(z^{-1}+m_{1}+z\right)
\end{aligned}
$$

where $m_{1}=\left[\left(Q_{1}+b_{1}^{2}\right)+\rho\left(1+a_{1}^{2}\right)\right] /\left(Q_{1} b_{1}+\rho a_{1}\right), \quad \sigma_{1}^{2}=\left(Q_{1} b_{1}+\rho a_{1}\right) / \alpha_{1}$. Let $\alpha_{1}$ denote the solution $\left(\alpha_{1}>1\right)$, then $\alpha_{1}=\left[m_{1} \pm\left(m_{1}^{2}-4\right)^{1 / 2}\right] / 2$, then $D_{c}\left(z^{-1}\right)=$ $\sigma_{1}\left(\alpha_{1}+z^{-1}\right), \sigma_{1}>0$. Consider the limiting positions of the zeros of $D_{f}$ and $D_{c}$. If $\mu=0, \alpha=-1.428$, or if $\mu \rightarrow \infty$ then $\alpha=-1.053$. Similarly, if $\rho=0, \alpha_{1}=2$, or if $\rho \rightarrow \infty$ then $\alpha_{1}=-1.053$. The polynomials $A$ and $B$ are relatively prime and a unique solution for the $L Q G$ optimal controller then follows, using Equation (1.86), with $n_{h_{0}}=n_{b}-1=2$ and $n_{g_{0}}=n_{a}-1=0$ : That is, $A H_{0}+B G_{0}=D_{c} D_{f}$ where,

$$
\left(1+a_{1} z^{-1}\right)\left(h_{0}+h_{1} z^{-1}+h_{2} z^{-2}\right)+z^{-2}\left(1+b_{1} z^{-1}\right) g_{0}=\sigma \sigma_{1}\left(\alpha+z^{-1}\right)\left(\alpha_{1}+z^{-1}\right)
$$

The general solution of this equation follows as:

$h_{0}=\sigma \sigma_{1} \alpha \alpha_{1}, h_{1}=a_{1} h_{0}+\sigma \sigma_{1}\left(\alpha+\alpha_{1}\right), h_{2}=\left(-a_{1} h_{1}+\sigma \sigma_{1}\right) /\left(1-a_{1} / b_{1}\right), g_{0}=-a_{1} h_{2} / b_{1}$

and in terms of the general system parameters the controller:

$$
C_{0}=g_{0} /\left(h_{0}+h_{1} z^{-1}+h_{2} z^{-2}\right)
$$

Equations (1.109)-(1.114) provide a mechanism to compute the $H_{2} / L Q G$ controller for a given system model of the assumed structure. They may therefore be used to construct an LQG explicit self-tuning controller [56, 57, 76].

Example 1.5.2 Numerical computation of the $H_{2} / L Q G$ controller

Consider the previous example, where the control $(\rho)$ and measurement noise $(\mu)$ weightings are set to unity. Thus, if $\rho=\mu=1$ then $m=-2.05606, m_{1}=6.5738$, $\alpha=-1.26645, \alpha_{1}=6.418, \sigma=1.14142, \sigma_{1}=0.40448$ and thence $h_{0}=-3.75258$, $h_{1}=-1.18658, h_{2}=-0.45124$ and $g_{0}=-0.21434$. The $H_{2} / L Q G$ controller in this case therefore becomes:

$$
C_{0}\left(z^{-1}\right)=0.214 /\left(3.753+1.187 z^{-1}+0.4512 z^{-2}\right)
$$

Example 1.5.3 $L Q G$ optimal control of coke ovens

The following example is based upon the application of $L Q G$ control in a self-tuning system for a coke oven battery heating system. The problem was described by Yan De-kun of the Xinjiang Institute of Physics, Chinese Academy of Science [73], and very good results were reported. A coke oven battery involves a huge mass and large heat expenditure (the oven consumes more than 30000 million calories of heat per hour in 
normal production). To economise on the usage of gas, to improve the quality of coke produced and to reduce air pollution, smooth and accurate control of the heating process is required. The temperature control technique must be able to compensate for changes of raw materials, fuel, operating conditions and environment. The temperature can be controlled via the supply of heating gas and air and must remain within allowable limits. The gas flow regulating valve is placed in the general distribution pipe, but the gas flow of each combustor is regulated by the operators with distribution valves. The combustor consists of the combustion chamber, fuel injection apparatus and igniter.

The air supply is determined by which doors are opened, and can be considered to be fixed in a given coking cycle. The suction is determined by the blocking boards which are closed on the branched flues. The pressure at the top of the heat store room and at the five observation holes must be maintained within predetermined limits. The manual control practice involved sampling the average combustor temperature every four hours. The pressure in the general gas distribution pipe and the suction in the branched flues was then regulated by the operators using their previous experience.

Thermocouples cannot be inserted in the combustors or separate wells. However, there is a correlation between the temperature of the gases in the heat storeroom tops and the temperatures of the combustors. Thus, the computer control must determine the envelope of the average temperature of the heat storeroom tops so as to indirectly control the average temperature along the coke oven battery.

The open-loop transfer for the temperature-regulating loop consists of the following cascaded subsystems:

(a) Coke heating temperature system with output the average temperature $T$.

(b) PI (proportional-integral) gas flow regulating loop which reduces difficulties caused by nonlinear valve characteristics.

(c) Fixed heat input temperature control system with control input $\{u(t)\}$.

The response of the system has a time constant of about $10 \mathrm{~h}$, suggesting a suitable sampling interval of $1 \mathrm{~h}$. Some of the characteristics of the system to be allowed for in design include:

(i) Time-varying: due to variability in raw materials, environmental conditions and degradation of components.

(ii) Nonlinearities: due to actuator nonlinearities and limitations of the instrumentation, and through the nonlinear relationship between the input and temperature output.

(iii) Disturbances: constant disturbances arise through errors in computing the balance point for the system and due to biases in the measurement system. Stochastic disturbances arise through the fluctuations in atmospheric and main pipe pressure, through the variations in wind direction and the changes in the temperature and composition of the gas.

(iv) System model: can vary between minimum (normal) and nonminimum-phase behaviour according to operating conditions 
System description: the plant model for the coke oven battery can be approximated by the model:

$$
W\left(z^{-1}\right)=\frac{B\left(z^{-1}\right)}{A\left(z^{-1}\right)}=z^{-1} \frac{\left(b_{0}+b_{1} z^{-1}+b_{2} z^{-1}\right)}{\left(1+a_{1} z^{-1}+a_{2} z^{-1}\right)}
$$

The disturbance model, assuming the reference is null, can also be assumed to be second-order and to be of the form:

$$
W_{d}\left(z^{-1}\right)=\frac{D_{f}\left(z^{-1}\right)}{A\left(z^{-1}\right)}=\frac{\left(d_{0}+d_{1} z^{-1}+d_{2} z^{-2}\right)}{\left(1+a_{1} z^{-1}+a_{2} z^{-2}\right)}
$$

The measurement noise $\{v(t)\}$ and reference $\{r(t)\}$ signals can be assumed to be null, so that $y(t)=z(t)$ in Figure 1.4. The variance of the process noise can be assumed to be scaled to unity.

Model for computations: the coke oven heating system employed an adaptive $L Q G$ solution. However, for the present purposes the controller will be computed for one particular estimated plant model at time $t=20 \mathrm{~h}$. The estimated polynomials can be listed as: $A\left(z^{-1}\right)=1-1.3529 z^{-1}+0.3941 z^{-2}, \quad B\left(z^{-1}\right)=z^{-1}\left(9.8275-0.8856 z^{-1}-\right.$ $\left.3.1579 z^{-2}\right), D_{f}\left(z^{-1}\right)=\left(1-0.2155 z^{-1}+0.1581 z^{-2}\right) / 8.6$. The zero-frequency gain of the plant model $W(1)=B(1) / A(1)=140.38$.

Cost function: the average power in the system, as defined by the cost index $J$, is to be minimised where $q, r>0$ are constants and the criterion: $J=E\left\{q e^{2}(t)+r u^{2}(t)\right\}$. For a scalar cost function one of the weights $q$ or $r$ can be set to unity, since it is only the relative magnitudes which affect the optimal controller. Thus, let $q=1$.

Choice of cost function weighting scalar $r$ : as a rule of thumb the speed of response of a system can normally be increased by a factor of ten under closed-loop control. If the plant transfer $W=A^{-1} B$ has an open-loop time constant of $10 \mathrm{~h}$, the first choice of $r$ can be to obtain a closed-loop time constant of approximately $1 \mathrm{~h}$. A further rule of thumb, to provide a starting value for the ratio of $Q_{c} / R_{c}=q / r$ is to let $W^{2}(1) q / r=100$. If, say, $q=1$ and $r=500$ then $W^{2}(1) q / r=40$. To check if the chosen weights are likely to provide the desired closed-loop response the frequency responses of $W^{*} Q_{c} W$ and $R_{c}$ shown in Figure 1.5, can be considered. As discussed in Chapter 6 the crossing point of the frequency responses will be close to the closed-loop dominant frequency (reciprocal of the dominant closed-loop time constant) or bandwidth point. From Figure 1.5 the crossing point $\omega_{c}=0.6 \mathrm{rad} / \mathrm{s}$. The values of $q=1$ and $r=500$ provide a good starting point for design.

Calculation of the controller: To compute the $L Q G$ optimal controller Equations (1.84) and (1.85) must be solved (or equivalently (1.86) if $A$ and $B$ are coprime). Since $\operatorname{deg}\left(D_{c}\right)=2$ (from (1.76)), then from inspection of (1.84) and (1.85) $g=3$, and $\operatorname{deg}(F)=2, \operatorname{deg}(G)=1$ and $\operatorname{deg}(H)=2$. Thus, the calculations may be summarised as follows:

(i) Calculate the spectral factor $D_{c}$ using (1.78) to satisfy: $D_{c}^{*} D_{c}=A^{*} r A+B^{*} B$ and from a standard spectral factorisation algorithm: $D_{c}=27.927785-28.0154 z^{-1}+$ $5.94445 z^{-2}$ 


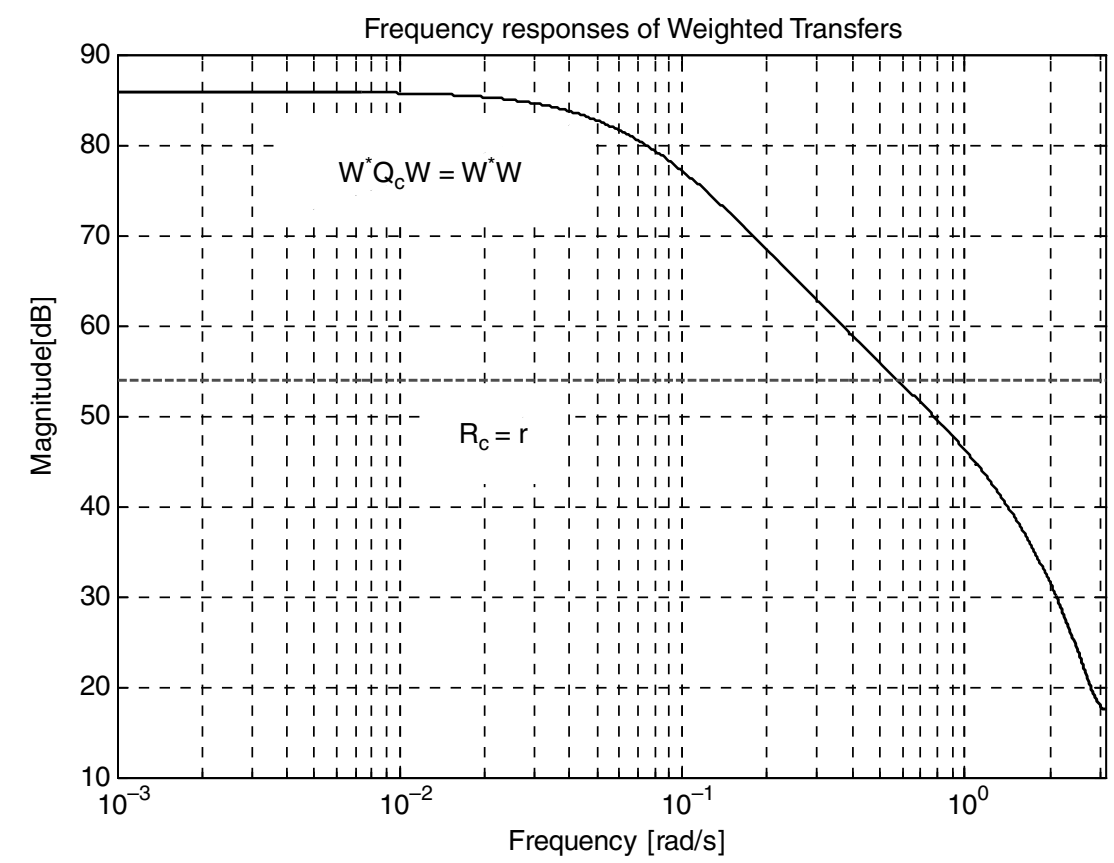

Figure 1.5 Solution of weighting gains to give desired cross-over frequency

(ii) Solve Equation (1.86): $A H+B G=D_{c} D_{f}$ using a Diophantine equation solver to obtain:

$$
H\left(z^{-1}\right)=3.2474-0.9325 z^{-1}-0.07696 z^{-2} \quad \text { and } \quad G\left(z^{-1}\right)=0.13926-0.04421 z^{-1}
$$

(iii) Compute the optimal controller: $C_{0}\left(z^{-1}\right)=H\left(z^{-1}\right)^{-1} G\left(z^{-1}\right)$ and implement the optimal control signal as:

$$
\begin{aligned}
u(t) & =-C_{0}\left(z^{-1}\right) y(t)=-\left(H\left(z^{-1}\right)^{-1} G\left(z^{-1}\right)\right) y(t) \\
& =-\left(g_{0} y(t)+g_{1} y(t-1)+h_{1} u(t-1)+h_{2} u(t-2)\right) / h_{0} \\
& =-(0.04288 y(t)-0.0136 y(t-1)-0.2872 u(t-1)-0.0237 u(t-2))
\end{aligned}
$$

Time-responses: the time-response of the plant is shown in Figure 1.6, together with the closed-loop response of the system. Note that the speed of response of the system has increased by the factor of 10 expected. The controller actually used in the self-tuning system was given by Yan De-kun [7] as:

$$
C_{0}\left(z^{-1}\right)=\left(0.1391-0.0442 z^{-1}\right) /\left(3.2469-0.9135 z^{-1}-0.0757 z^{-2}\right)
$$

that corresponds to that given above (allowing for numerical inaccuracies). 

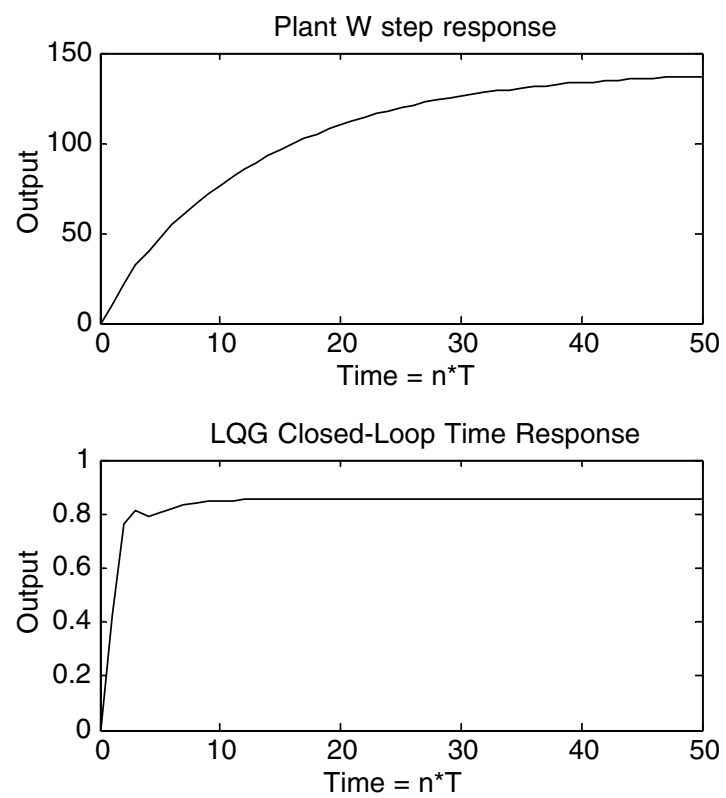

Figure 1.6 Unit step function responses of coke oven and $L Q G$ feedback system

Practical experience of implementation: the average heating required per hour is about $2 \%$ lower than with a well-tuned PID controller. The energy savings are significant and the $L Q G$ design adapts particularly well to changes in the system minimum/ nonminimum phase behaviour.

\subsection{Benchmarking}

There are many reasons why control loops on a system or process plant are poorly tuned and some of these include:

1. Engineers are often conservative in nature and they are cautious about changes to controllers that might lead to instability.

2. There are extreme time pressures on engineering staff so that if a loop functions, even poorly, it will often be accepted as being adequate.

3. Process plant engineers often do not have the level of experience or skill to use what might be considered advanced algorithms to gain real improvements that are feasible.

4. Engineers may not use gain scheduling techniques to cope with nonlinearities unless essential.

Over the last decade there have been rapid advances in the development of tools for control loop performance assessment and benchmarking. Performance assessment methods for control loops have in the past depended upon criteria such as minimum 
variance $(M V)$ cost indices. This type of measure of performance has been applied successfully in petrochemical and related applications, where product quality or economic performance could be related directly to the variance of the signal. However, for many real applications such a measure is unrealistic. This is partly because a minimum variance controller has poor characteristics and partly because it is of a high order for implementation. In either event such a controller would not be implemented in practice and hence its use for comparison purposes is suspect.

Harris showed that a lower bound on the closed-loop output variance could be obtained by analyzing routine closed-loop operating data [86, 104]. This provided a formal assessment tool with which to assess the behaviour of stochastic systems that did not require knowledge of formal models. Moreover, the $M V$ criterion is particularly appropriate for industrial processes, where the regulating behaviour can be tied to economic performance. The major disadvantage is that unrealistic control action arises when the control signal is not limited by a control costing term. Such a benchmark is therefore flawed in its basic form.

The $L Q G$ cost index provides a more realistic measure of performance than the minimum variance criterion which is often employed. The above expression (Equation 1.104) for the minimum cost is useful for benchmarking control loops when model information is available. The technique involves a comparison of the cost index evaluated for the controller in use (such as $P I D$ ), with the minimum possible cost, as represented by the minimum variance or the $L Q G$ minimum cost (Equation 1.104). The generalised minimum variance $(G M V)$ control law provides a similar, but alternative benchmark figure of merit and is closely related to $L Q G$ design. The GMV control philosophy has characteristics midway between the $M V$ and $L Q G$ design philosophies. Both methods provide real advantages for benchmarking, particularly in comparison with $M V$-based algorithms [102, 103, 105, 106].

The benchmarking cost indices provide useful insights into the performance of a controller. There are of course other more traditional measures of performance such as the mean square error, rise time and peak overshoot, but the $H_{2} / L Q G$ index is particularly attractive, since it is convenient algorithmically and can often be linked to process quality or energy minimisation requirements. If, of course, the benchmark cost index is computed for a real plant, but the benchmark is based on a full-order optimal controller and the result is poor, the question arises as to the reason. If, say, the classical controller on the plant is of low order this may explain the result or it may be that the controller is just poorly tuned.

\subsubsection{Restricted Structure Benchmarking}

New methods have recently been developed for benchmarking and tuning controllers of restricted structure $(R S)$. That is, a $R S$ controller can be optimised for an existing system structure. Such controllers might have, say, a multivariable $P I D$ or other classical form and are introduced in Chapter 6 and Section 6.10. The new benchmark figures of merit take into account the limitations of the existing system structure and are therefore more realistic. This is referred to as 'restricted structure performance assessment and benchmarking' and is a useful advance on previous work, where the benchmark figures may not have been actually achievable with in the limitations of the physical system. These $R S$ 
benchmarking methods use a more advanced criterion which requires additional information. They provide an indication of how well loops are tuned and the degree of improvement that may be possible.

The $R S$ benchmarking methods also enable those loops, which are important in complex multivariable systems to be determined, so that priorities can be established for tuning. A typical process plant may have 500-5000 control loops and an important benchmarking problem is how to determine which loops are important to the product quality, or the economic performance of the system. This problem of so-called loop prioritisation involves the use of benchmarking algorithms to determine the critical loops in large multivariable interacting processes. The $R S$ benchmarking technique may also be used for structures assessment of multivariable systems which is related to the rather old ideas of dynamic gain array for structures assessment.

This subject of benchmarking is, of course, independent of the problem of actually tuning such systems. Once a system has been demonstrated to be underperforming, there are various options available. One possibility is to introduce new control equipment vendors or suppliers, but another is to consider the use of some of the improved tuning techniques now available. The tuning of an existing control system with a given structure and limitations on equipment is rather different from a new design where there are no limits on controller structure or order. A technique will be described later for tuning such systems to satisfy $\mathrm{H}_{2}$ or even $H_{\infty}$ criteria. The minimum variance, $G M V$ and $L Q G$ family of benchmarking criteria are very suitable for the continuous process industries but servo systems and batch processes require new algorithms, possibly based on finite-time $L Q$ or even $H_{\infty}$ methods. This type of process and some continuous processes can involve significant nonlinear behaviour and new benchmarking methods for nonlinear systems, based on results from Chapter 11, may also be postulated.

Benchmarking techniques were developed only for assessing controllers, but they can be used as an aid to tuning controllers or in a modified form for plant diagnostics. There is growing industrial interest because of the competitive pressures between companies and a rapidly developing body of knowledge [90-94] in the area. There remain important problems to consider, such as benchmarking batch processes and benchmarking the control of grade transitions. Since many of the benchmarking results only depend upon normal operating records, such techniques have been proposed as a way of condition monitoring control loops in addition to their use in more traditional benchmarking applications.

\subsubsection{Rules for Benchmark Cost Function Selection}

There follows below some general guidelines on which cost function should be employed for different types of application. Some of the common features of the techniques used are as follows:

1. They are appropriate for systems with continuous disturbances or reference changes.

2. They are mostly applicable to process plants that have continuous operation.

3. The techniques mostly assume the system is linear in the region of operation. 
There are a number of different algorithms referred to above and some guidelines for use of the most popular follow.

Minimum variance: the minimum variance benchmarking method is the simplest and should be used under the following circumstances:

1. Where only a rough guideline is needed and simplicity is at a premium.

2. Where variations of control signals and actuator movements are not so important.

3. Where very tight control of the process output is demanded without concerns for power or energy used.

The circumstances where minimum variance control should not be used are as follows:

1. If in practice there are tight limits on actuator movements and control signal variations.

2. If the controller is in practice restricted to being low order and of a particular form.

3. When the cost involved is not simply an output variance or tracking error.

Generalised minimum variance benchmarking: the generalised minimum variance $(G M V)$ benchmarking should be used for the following situations:

1. If control actuator movements have to be limited.

2. When costs of operation involve a mixture of performance and energy requirements.

3. When control power must be limited due to actuator drives or power supplies.

The GMV benchmarking should not be used when simplicity is needed or in the following situations

1. When the actual controller to be benchmarked is of low order or has other structural limitations.

2. When the cost function is naturally a sum of squares rather than a square of sum form.

Restricted structure LQG benchmarking: the linear quadratic gaussian (LQG) benchmark is very suitable for more sophisticated applications and particularly when low-order or restricted structure controllers are employed. That is, controllers to be benchmarked that already have a well-defined structure such as a PID controller. The RS benchmarking technique and a suitable algorithm is discussed later in Section 6.10.6.

The situations where LQG RS Benchmarking should be used may be listed as follows:

1. When cost functions involve a sum of squared energy and performance terms.

2. When the controllers to be used in practice are of a restricted structure. 
The restricted structure $(R S)$ benchmarking should not be used in the following situations.

1. Where simplicity of the benchmarking algorithm is paramount.

2. Where engineers do not wish to spend the time selecting the performance or cost index.

\subsection{Condition Monitoring}

The new generation of control loop performance assessment and condition monitoring tools were produced over the last decade, mainly for use in the chemical and petrochemical industries. Very recent advances make such techniques available for faster systems, as found in metal processing applications. The main benefit of the new condition monitoring approach is that it mainly requires normal operating records rather than detailed models of processes. It is a small step to use similar techniques to those for benchmarking for condition monitoring applications. Almost the same software may be used as a 'condition monitoring' aid to determine the onset of unusual conditions, representative of a build-up of possible faults or breakdown.

The approach relies upon the calculation of performance indicators, which are defined so that good control loop performance corresponds with the best quality control or economic performance. This is an advance on statistical process control (SPC), since the indicators can also be used for optimisation purposes. That is, the benchmarks can provide an aid to control systems tuning to optimise the performance indicators. Thus, the performance metrics can be used to sense possible faults and provide a criterion for tuning the optimal system.

The new benchmarking and condition monitoring software tools being produced can be utilised in different ways:

- to assess the performance of an existing system and determine which loops need tuning;

- to quantify the degree of improvement that is possible;

- to provide a basis for optimising new installations with complex interaction dynamics;

- to provide process operators with indicators of control system performance so that possible fault conditions can be detected, either visually or automatically.

There are many approaches to condition monitoring and fault detection and many of these require very detailed models of the process and require Kalman or $H_{\infty}$ estimators to detect the onset of fault conditions. Unfortunately, such detailed knowledge is not really available in the process industries, although it may be possible in the aerospace industry. Another approach is to use neural networks to estimate model structures and model parameters but they do have unpredictable behaviour on occasions. The statistical process control methods may also be used to interogate data and to predict the onset of possible fault conditions. However, this approach does not provide clear indicators by which the best performance can be judged. The recent ideas referred to above develop condition monitoring procedures from a different historical perspective. That is from the 
move over the last decade to produce performance and benchmarking techniques to judge the performance of control loops. Such methods calculate performance measures, such as the variance of the tracking error, and these are then used to judge how well an existing system is behaving.

The benefits of new condition monitoring algorithms based on the benchmarking ideas may be summarised as:

1. Some of the performance metrics do not need detailed model information, but require only normal operating records for computation.

2. The same performance metrics may be used as a measure of performance of the systems behaviour and then used for optimisation purposes.

3. These performance measures may be used in a rather naive way to provide display of quality normalised between 0 and 1 , or in a more sophisticated way, where an investigator can trace the origins of faults from the multiplicity of indices which can be computed.

4. The approach provides new tools for consultancy companies and methods of in-house analysis and optimisation for manufacturing companies.

\subsection{Combining $\mathrm{H}_{2}, \mathrm{H}_{\infty}$ and $\ell_{1}$ Optimal Control Designs}

There is often a need to have a combination of useful properties of the $H_{2}$ and $H_{\infty}$ design and synthesis philosophies. Many contributions to the literature are now occurring in this area, based upon the state-space approach [47-55]. A quantitative comparison between $H_{2}$ and $H_{\infty}$ performance has been performed by Zhou [70]. However, there has been little work on $\mathrm{H}_{2} / \mathrm{H}_{\infty}$ optimal control via the polynomial systems approach, other than that reported in Section 3.8.2. It is very likely that in future years $H_{2}$ and $H_{\infty}$ optimal control will be thought of as special cases of a general $H_{2} / H_{\infty}$ theory. The seeds of such a general theory can be found in the links established between the two cases presented in the following.

Dahleh and Pearson [41, 42] considered the problem of minimising the $L_{1}$ (continuous-time) or $\ell_{1}$ (discrete-time) norms of the closed-loop impulse response. This is particularly of value when the maximum amplitude of the output of a system must be limited, when the inputs are arbitrary bounded functions, such as step functions, sinusoids of unknown frequencies, etc. [65]. These problems may be solved using simple linear programming techniques [85].

In continuous-time $H_{\infty}$ optimisation, the objective is to minimise the worst case error that can occur in the operation of a system. The error is measured by the $L_{2}$ norm and the system inputs are constrained to have finite energy (bounded $L_{2}$ norms). In practice disturbances are often persistent, that is, they act continuously over time and do not belong to the class of signals in $L_{2}$. Thus, there is an argument for considering a different mathematical setting. In $L_{1}$ optimisation the induced norm on the operator $M$ is given by:

$$
\|M\|_{1}=\sup _{f \neq 0} \frac{\|M f\|_{\infty}}{\|f\|_{\infty}}
$$


and minimising the $L_{1}$-norm of the closed-loop impulse response limits the $L_{\infty}$ norm of the system outputs, given $L_{\infty}$ bounded inputs. Since disturbances which are of bounded amplitude are common, the $L_{1}$ optimisation problem can be motivated physically.

Liu and Zakian [58-60] have introduced the term sup regulator for use in so called critical systems. A critical system is such that the absolute output of the system must be limited to a prescribed bound when the input is driven by a random disturbance, where there is a bound on the mean value of the increment. The analysis presented by these authors uses a polynomial framework and requires the solution of a Diophantine equation. Controllers of this type can therefore be derived using the same type of solution and results, to follow for $H_{2} / L Q G$ and $H_{\infty}$ designs. The approach will not be discussed further here since at the present time the results appear to be somewhat less attractive for general industrial applications, but it may be an area for development in adaptive control [61].

\subsection{Linear Matrix Inequalities}

Linear matrix inequalities (LMIs) have been known for many years, but a milestone occurred in 1940 when Lure and Postnikov and others in the Soviet Union applied Lyapunov methods to nonlinear control problems. The stability criteria had the form of LMIs. The next development came in the early 1960s when Yakubovich, Popov, Kalman and others succeeded in reducing the solution of the LMIs from the work of Lure to simple graphical criteria using the positive real lemma. It was known by 1970 that the LMI appearing in the positive real lemma can be solved not only by graphical means, but also by solving a certain algebraic Riccati equation [95]. A milestone in LMI history was the seminal paper by J.C. Willems [96]. In this paper the equivalence between a certain frequency domain inequality $(F D I)$ and a corresponding $L M I$ was established. This equivalence makes it possible to describe bounds on a transfer function as an $L M I$ via the bounded real lemma. Willems made the observation that such an LMI can be formulated as a convex optimisation problem. These results, together with the recently developed efficient algorithms for numerically solving convex $L M I$ problems, lead to a powerful framework for solving general multi-objective numerical problems for linear systems.

Recall that a linear matrix inequality is any constraint that can be written in the canonical from:

$$
A(x)=A_{0}+x_{1} A_{1}+\cdots+x_{n} A_{n}<0
$$

where $x \in R^{n}$ is a vector of unknown scalars $x_{1}, \ldots, x_{N}$ (called the decision variables) and $A_{0} \ldots A_{N}$ are given symmetric matrices. The $L M I$ is a convex constraint on $x$ since $A(y)<0$ and $A(z)<0$ imply: $A((y+z) / 2)<0$. Convexity is an important property, since if a given problem is convex, then it can be solved numerically with a guarantee of finding a solution when one exists. This is true even if the problem has no analytical solution. It is of course a very desirable property and leads to the observation that in optimisation problems the crucial property is not linearity (versus nonlinearity) but convexity (versus nonconvexity), [97].

A solvable convex optimisation problem has a set of solutions called the feasible set. The feasible set is a convex subset of $R^{n}$, that is, if $x_{1} \in R^{n}$ and $x_{2} \in R^{n}$ are solution to the 
problem, then $x_{3}=\left(x_{1}+x_{2}\right) / 2 \in R^{n}$ is also a solution. A system of $L M I$ constraints can be regarded as a single $L M I$ since: $B_{1}(x)<0, \ldots, B_{K}(x)<0$ is equivalent to: $B(x)=\operatorname{diag}\left(B_{1}(x), \ldots, B_{K}(x)\right)<0$. In most control applications LMIs arise in the form $\mathcal{L}\left(x_{1}, \ldots, x_{N}\right)<\mathcal{R}\left(x_{1}, \ldots, x_{N}\right)$ where $\mathcal{L}$ and $\mathcal{R}$, are affine functions of structured matrix variables. The well known $L M I$ toolbox can handle any systems of LMIs of the general form: $\mathcal{N}^{\mathcal{T}} \mathcal{L}\left(X_{1}, \ldots, X_{L}\right) \mathcal{N}<\mathcal{M}^{T} \mathcal{R}\left(X_{1}, \ldots, X_{N}\right) \mathcal{M}$ where $\left(X_{1}, \ldots, X_{L}\right)$ are structured matrix variables. The $N$ and $M$ are the left and right outer factors, respectively and are of identical dimension. The above $L M I$ can be written: $A(x)<0$. Finding a solution to such an inequality is called a feasibility problem. The toolbox also contains an equation solver for the linear objective minimisation problem of the form:

$$
\min _{B(x)<0} c^{T} x
$$

which can be used for multi-objective design problems.

\subsection{Concluding Remarks}

The investment required in control systems is often small relative to that needed for mechanical, chemical, electrical plant and equipment, but it is still significant. The control system assets (Figure 1.7), must be utilised to the full if companies are to remain competitive and this requires the most effective control techniques to be applied. In many cases the simplest solution is best, but in some applications more sophistication is needed and a range of techniques must be available. The benchmarking and performance assessment methods referred to provide methods for assessing the most suitable controls and tuning.

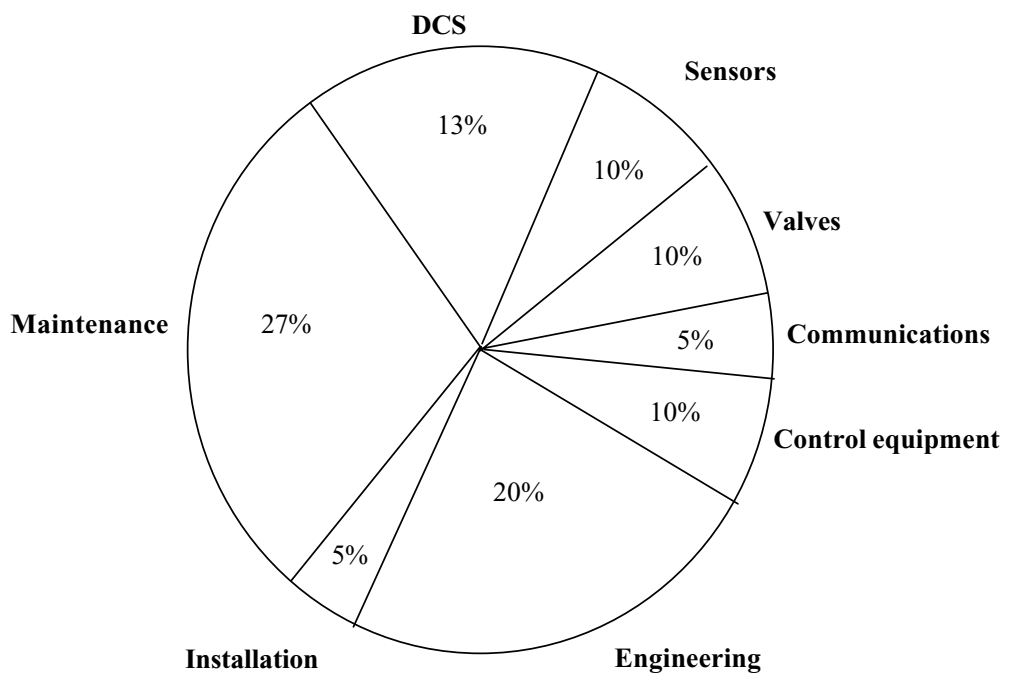

Figure 1.7 Control loop assets 
The $H_{2} / L Q G$ and $H_{\infty}$ optimal control problems have both been introduced and it will be shown later that $H_{\infty}$ robust design is a multivariable control design procedure, which can enable good performance and stability properties to be assured, even when the system contains uncertainties and modelling errors. There is an inexorable trend towards the tailored commercial software packages for the solution of standard industrial control problems. The polynomial equations which arise in the following can be easily programmed in commercial packages, since most of the steps in the algorithms are available as standard commands.

Both $H_{2} / L Q G$ and $H_{\infty}$ controller design approaches have a place in the toolkit of the control engineer. The class of problems considered for $H_{\infty}$ and $H_{2} / L Q G$ designs is different and the $H_{\infty}$ philosophy is particularly appropriate when improving robustness, in the face of plant perturbations, or parameter uncertainty. The $H_{\infty}$ controllers are more difficult to calculate, but special forms of the controller suitable for use in industrial systems are sufficiently simple for practical industrial usage. Different versions of $H_{\infty}$ designs offer different advantages. The $H_{\infty}$ equivalent of minimum variance controllers, generalised minimum variance controllers, predictive controllers and $L Q G$ controllers have also been defined. The $H_{\infty}$ version of the generalised predictive control $(G P C)$ law in polynomial systems form was developed by Bentsman and co-workers [75]. There are further opportunities for the development of learning, robust adaptive and self-tuning controllers using these $H_{\infty}$ control design methods.

The modelling and control paradigm to be used in the following is based upon a polynomial systems representation and both $H_{2}$ or $H_{\infty}$ optimisation. The frequency domain polynomial system approach has great applications potential in learning, adaptive and nonadaptive systems. Although a number of texts have introduced the system theory aspects of the polynomial systems approach [17, 33-35], few have discussed the applications problems. The following concentrates on providing the solutions of the different control and signal processing problems, for applications. Numerical algorithms are not considered in detail, since the basic routines are available in commercial packages. The $L Q G / H_{2}$ design procedures have also been modified, learning from the $H_{\infty}$ robust design results. What might be termed a robust $L Q G / H_{\infty}$ design philosophy has emerged. This approach depends upon the choice of dynamic cost function weightings to improve robustness, in much the same way as in the $H_{\infty}$ design method.

Although further analysis and design procedures will no doubt evolve, the $H_{\infty}$ approach seems to be one of the most promising to emerge in the last two decades. It appears that $H_{\infty}$ optimisation, possibly linked to $H_{2}$ minimisation requirements, provides a sound theoretical framework on which to build computer friendly and practical industrial design procedures.

\subsection{Problems}

Problem 1.1 Computation of the $H_{\infty}$ norm. Consider the transfer-function matrix for a continuous-time system: $G(s)=\bar{C}(s I-\bar{A}) \bar{B}$ where $(\bar{A}, \bar{B}, \bar{C})$ denotes a state-space triple, and define the $2 n \times 2 n$ matrix:

$$
H=\left[\begin{array}{cc}
\bar{A} & \overline{B B}^{T} \\
-\bar{C}^{T} \bar{C} & -\bar{A}^{T}
\end{array}\right]
$$




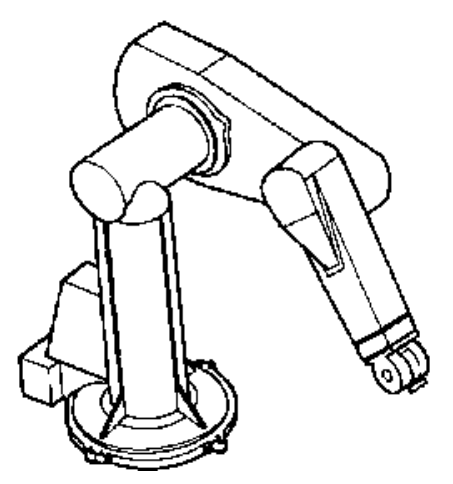

Figure 1.8 PUMA 500 manipulator

Show that $\|G(s)\|_{\infty}<1$ if and only if $H$ has no eigenvalues on the imaginary axis. Suggest a method of computing the $H_{\infty}$ norm of $G(s)$ based upon this result.

Problem 1.2 Youla parameterisation. Consider a plant represented in polynomial form: $W=A^{-1} B$ and a controller parameterised as: $C_{0}=(X-B K)^{-1}(Y+A K)$ where $X$ and $Y$ are polynomials which satisfy: $A X+B Y=I$ and $K$ denotes a stable transfer function. Show that the three possible closed-loop transfer functions are simple affine functions of $K$ (an affine function of $K$ has the form $T_{1}+T_{2} K T_{3}$ ), and that such a system is stable for all stable $K$.

Problem 1.3 $L Q G$ control of a robot arm. Consider the control of the joint angular position of a PUMA 500 robot arm, shown in Figure 1.8, which has six degrees of freedom. Identifying the system polynomials, with a sampling interval of $T_{s}=0.1 \mathrm{~s}$, at various operating points [74]:

$$
\begin{aligned}
A\left(z^{-1}\right) & =1+a_{1} z^{-1}+a_{2} z^{-2}+a_{3} z^{-3}, B\left(z^{-1}\right)=z^{-1}\left(b_{0}+b_{1} z^{-1}+b_{2} z^{-2}\right), C_{d}\left(z^{-1}\right) \\
& =1+c_{1} z^{-1}+c_{2} z^{-2}
\end{aligned}
$$

gives the coefficients in Table 1.2.

Table 1.2 The estimated plant parameters at various operating points

\begin{tabular}{ccccccccc}
\hline Angle $(\mathrm{rad})$ & $a_{1}$ & $a_{2}$ & $a_{3}$ & $b_{0}$ & $b_{1}$ & $b_{2}$ & $c_{1}$ & $c_{2}$ \\
\hline-0.50 & -2.8 & 2.6 & -0.8 & 0.0205 & 0.003 & -0.0185 & 0.95 & 0.15 \\
0.00 & -2.9 & 2.7 & -0.9 & 0.0200 & 0.001 & -0.0200 & 0.94 & 0.12 \\
0.30 & -3.0 & 2.6 & -1.0 & 0.0200 & 0.002 & -0.0190 & 0.61 & 0.39 \\
1.57 & -2.9 & 2.9 & -0.9 & 0.0200 & 0.001 & -0.190 & 0.40 & 0.47 \\
2.00 & -3.00 & 2.9 & -1.0 & 0.0235 & 0.002 & -0.0210 & 0.50 & 0.56 \\
3.00 & -2.8 & 2.4 & -0.8 & 0.0280 & 0.001 & -0.0180 & 0.20 & 0.40 \\
\hline
\end{tabular}


(i) Show that the system is nonminimum phase and open-loop unstable.

(ii) Consider the minimisation of the cost function (Equation 1.82) where $Q_{c}=I$ and $R_{c}=0.01$. Assume that the measurement noise variance is null and process noise variance is unity. Compute the $L Q G$ controllers for each of the operating points.

(iii) Find one controller which stabilises the system at all operating points and maximises robustness margins. Use open-loop frequency response plots to justify choice of $L Q G$ cost and controller.

Problem 1.4 Coke oven temperature control. Compute the $H_{2} / L Q G$ controller for the coke oven where $a=A$ and $b=B$ in Example 1.5.3. Let the disturbance numerator polynomial $c=C=1$ and assume the variances of the white noise signals are unity. Thence the white measurement noise model $c_{n} / a_{n}=1$ or $c_{n}=1, a_{n}=1$. Calculate the controller: $C_{0}=C_{0}\left(z^{-1}\right)=C_{0 d}\left(z^{-1}\right)^{-1} C_{0 n}\left(z^{-1}\right)$, for the cost weightings used in the example. That is, $b_{q} / a_{q}=1$ and $b_{r} / a_{r}=0.1^{1 / 2} \Rightarrow b_{q}=a_{q}=1$ and $b_{r}=0.1^{1 / 2}$ and $a_{r}=1$.

Problem 1.5 Minimum variance control

(a) Consider an ARMAX model for a stochastic system:

$$
y(t)=\left(B\left(z^{-1}\right) / A\left(z^{-1}\right)\right) u(t)+\left(C\left(z^{-1}\right) / A\left(z^{-1}\right)\right) \zeta(t)
$$

where $\{\zeta(t)\}$ denotes a zero mean white noise sequence. Derive the expression for the minimum variance control law to minimise the variance of the output: $y(t)$, working from first principles and using the Diophantine equation: $E_{0}\left(z^{-1}\right) A\left(z^{-1}\right)+z^{-k} F_{0}\left(z^{-1}\right)=C\left(z^{-1}\right)$ where $E_{0}$ and $F_{0}$ are polynomials in the unit delay operator $z^{-1}$ and $C\left(z^{-1}\right)$ is minimum phase.

(b) Describe the limitations of this control law by listing the disadvantages for practical applications.

(c) In what types of industry might an improved minimum variance control law be used. Describe a possible application and explain why such controllers are valuable.

Problem 1.6 Scalar submarine design problem. Consider a pitch control $H_{\infty}$ design problem for a submarine where the system and weightings are placed in a standard system form. Compute the state-space form of the $H_{\infty}$ output feedback controller if the standard system matrix, for 13 states, 3 outputs and 5 inputs, is as shown below. Let the system model be:

$$
P=\left[\begin{array}{ll}
\bar{A} & \bar{B} \\
\bar{C} & \bar{D}
\end{array}\right]
$$


where the system matrices are:

$$
\bar{A}=\left[\begin{array}{cccccccccc}
0 & 0 & 0 & 0 & 0 & -0.02 & 0 & 0 & 0 & 0 \\
0 & 0 & 1 & 0 & 0 & 0 & 0 & 0 & 0 & 0 \\
0 & 0 & 0 & 1 & 0 & 0 & 0 & 0 & 0 & 0 \\
0 & 0 & 0 & 0 & 1 & 0 & 0 & 0 & 0 & 0 \\
0-1.8099 \times 10^{-3} & -0.040613 & -0.251864 & -0.367245 & 0 & 0 & 0 & 0 & 0 \\
0 & 0 & 0 & 0 & 0 & -10^{-4} & 0 & 0 & 0 & 0 \\
0 & 0 & 0 & 0 & 0 & 0 & 0 & 1 & 0 & 0 \\
0 & 0 & 0 & 0 & 0 & 0 & -100 & -20 & 0 & 0 \\
0 & 0 & 0 & 0 & 0 & 0 & 0 & 0 & -0.038 & 0.89604 \\
0 & 0 & 0 & 0 & 0 & 0 & 0 & 0 & 1.71 \times 10^{-3} & -0.091976 \\
0 & 0 & 0 & 0 & 0 & 0 & 0 & 0 & 1 & 0 \\
0 & 0 & 0 & 0 & 0 & 0 & 0 & 0 & 0 & 1 \\
0 & 0 & 0 & 0 & 0 & 0 & 0 & 0 & 0 & 0
\end{array}\right]
$$

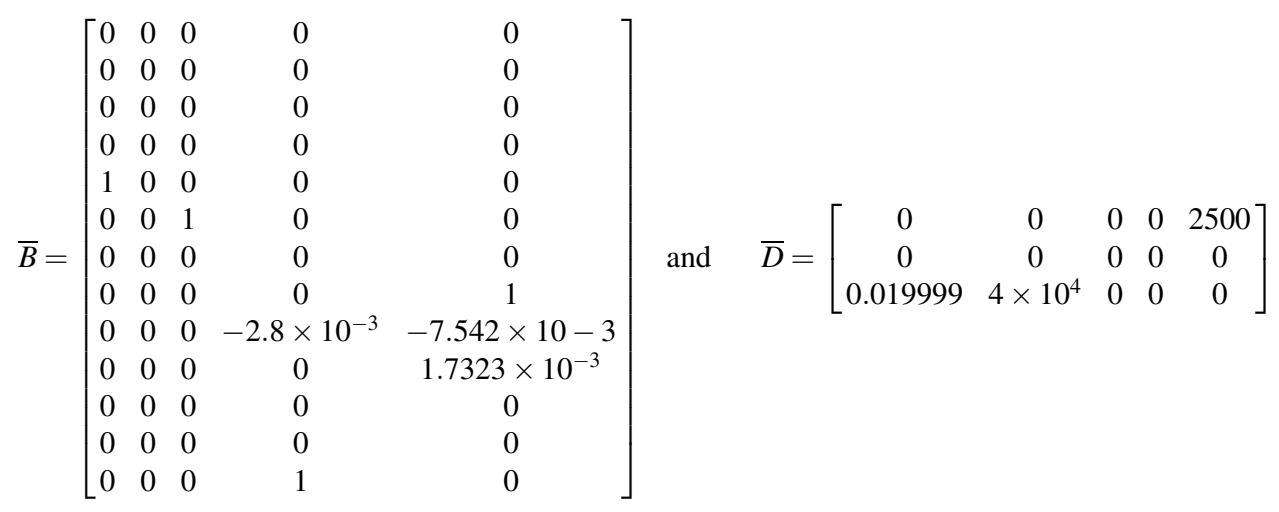

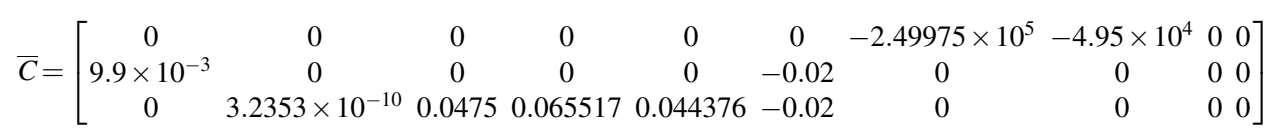

\subsection{References}

1. Nyquist H., 1932, Regeneration Theory Bell System Tech.

2. Bode H.W., 1945, Network Analysis and Feedback Amplifier Design, D. Van Nostrand Company, Inc., Princeton, N.J.

3. Nichols N.B., 1954, Backlash in a velocity lag servomechanism, Trans. ALEE, 22(2), 173-179.

4. Evans W.R., 1954, Control System Dynamics, McGraw Hill Book Company Inc., New York.

5. Horowitz I., 1991, Survey of quantitative feedback theory (QFT), International J. Control, $\mathbf{5 3}$ (2), 255-291.

6. Wiener N., 1949, Extrapolation, Interpolation and Smoothing of Stationary Time Series, with Engineering Applications, New York Technology Press and Wiley (Originally issued in Feb. 1942 as a classified National Defence Research Council Report). 
7. Kalman R.E., 1960, A new approach to linear filtering and prediction problems, Journal of Basic Engineering, 82, 35-45.

8. Safonov M.G. and Athans M., 1977, Gain and phase margin for multiloop $L Q G$ regulators, IEEE Trans. Auto. Control, AC-22, 2.

9. Rosenbrock H.H., 1974, Computer-aided Control System Design, Academic Press.

10. MacFarlane A.G.J., 1971, Linear multivariable feedback theory: a survey, IFAC Symposium on Multivariable Control Systems, Dusseldorf.

11. Mayne D.Q., 1973, The design of linear multivariable systems, Automatica, 9, 201-207.

12. Zames G., 1979, Feedback and optimal sensitivity: model reference transformation, weighted seminorms, and approximate inverses, Proc. 17th Allerton Conference, 744-752.

13. Zames G., 1981, Feedback and optimal sensitivity: model reference transformations, multiplicative seminorms and approximate inverses, IEEE Trans. Auto. Control, AC-26, 301-320.

14. Grimble M.J., 1987, $H_{\infty}$ robust controller for self-tuning control applications, Part I: Controller design, Int. J. Control, 46, (4), 1429-1444.

15. Fairbairn N.A. and Grimble, M.J., $1990, H_{\infty}$ robust controller for self-tuning applications, Part 3: self-tuning controller implementation, Int. J. Control, 52, (1), 15-36.

16. Prime H., 1969, Modern Concepts in Control Theory, McGraw-Hill, pp. 140-142.

17. Kucera K., 1979, Discrete Linear Control, John Wiley and Sons, Chichester.

18. Kwakernaak H., 1986, A polynomial approach to minimax-frequency domain optimization of multivariable feedback systems, Int. J. Control, 117-156.

19. Duren P.L., 1970, Theory of HP Spaces, Academic Press, London.

20. Hardy G.H., 1915, The mean value of the modulus of an analytic function, Proc. London Math. Soc., 14, 269-277.

21. Zames G. and Francis B.A., 1981, A new approach to classical frequency methods feedback and minimax sensitivity, IEEE Conf. on Dec. and Control, San Diego, 867-874.

22. Francis B.A., 1987, A Course in $H_{\infty}$ Control Theory, Springer Verlag, NY.

23. Doyle J.C., Glover K., Khargonekar P. and Francis, B.A., 1989, State-space solution to standard $\mathrm{H}_{2}$ and $\mathrm{H}_{\infty}$ control problems, IEEE Trans. Auto Control, 34, (8), 831-846.

24. Stoorvogel A., 1992, The $H_{\infty}$ Control Problem, a State Space Approach, Prentice Hall, London.

25. Yaesh I. and Shaked U., 1991, A transfer function approach to the problems of discrete-time systems: $H_{\infty}$ optimal linear control and filtering, IEEE Trans. Auto Control, AC, 36, (1), 1264 1271.

26. Basar T., 1989, A dynamic games approach to controller design: disturbance rejection in discrete-time, Proc. IEEE CDC Conf, Tampa, Florida, 407-414.

27. Mustafa D. and Bernstein D. S., 1991, $L Q G$ cost bounds in discrete-time $H_{2} / H_{\infty}$ control, Proc. Symposium Organised by Inst. of Meas. and Control on Robust Control System Design Using $H_{\infty}$ and Related Methods. P. Hammond (ed.), 295-307.

28. Iglesias P.A. and Glover K., 1991, State-space approach to discrete-time $H_{\infty}$ control, Int. $J$. control, 54, (3), 1031-1073.

29. Grimble M.J. and Johnson M.A., 1988, Optimal Multivariable Control and Estimation Theory: Theory and Applications, Vols I and II, Wiley, Chichester.

30. Petersen I.R., Anderson B.D.O. and Jonckheere E.A., 1991, A first principle solution to the non-singular $H_{\infty}$ control problem, Int. J. Robust Nonlinear Control, 2, 181-185.

31. Grimble M.J., 1986, Optimal $H_{\infty}$ robust controllers and the relationship to $L Q G$ design problems, Int. J. Control., 43, (2), 351-372.

32. Grimble M.J., 1987, $H_{\infty}$ robust controller for self-tuning control applications.: Part 2: Self tuning and robustness, Int. J. Control, 46, (5), 1819-1840.

33. Barnett S., 1971, Matrices in Control Theory, Van Nostrand Reinhold, London. p. 10. 
34. Wolovich W.A., 1974, Linear Multivariable Systems, Springer, New York.

35. Kailath T., 1980, Linear Systems, Prentice Hall: Englewood Cliffs, NJ.

36. Grimble M.J., 1990, $H_{\infty}$ controllers with a PID structure, J. Dynamic Systems Measurement and Control, 112, 325-336.

37. Grimble M.J., 1987, Relationship between polynomial and state-space solutions of the optimal regulator problem, Syst. Control Letters, 98, 411-416.

38. Furuta K. and Phoojaruenchanachai S., 1990, An algebraic approach the to discrete-time $H_{\infty}$ control problems, Proc. American Control Conf., San Diego, California.

39. Morari M., and Zafiriou E., 1989, Robust Process Control, Prentice Hall: Hemel Hemstead.

40. Owens D.H., 1978, Feedback and multivariable systems, IEE Control Engineering Series, Peter Peregrinus, Stevenage.

41. Dahleh M.A. and Pearson J.B., 1987, $\mathrm{L}_{\infty}$ optimal compensators for continuous-time systems, IEEE Trans. Auto. Control, AC-32, (10), 889-895.

42. Dhaleh M.A. and Pearson J.B., 1987, $\mathrm{L}_{1}$ optimal feedback controllers for MIMO discrete-time systems, IEEE Trans. Auto. Control, AC-02, (3), 314-321.

43. Kwakernaak H., 1984, Minimax frequency domain optimization of multivariable linear feedback systems, IFAC World Congress, Budapest, Hungary.

44. Kwakernaak H., 1985, Minimax frequency domain performance and robustness optimization of linear feedback systems, IEEE Trans. Auto. Control, AC-30, (10), 994-1004.

45. Kwakernaak H., 1990, The polynomial approach to $H_{\infty}$-optimal regulation, Lecture Notes, CIMIE Course on Recent Developments in $H_{\infty}$ Control Theory, Como Villa Olmo.

46. Kwakernaak H., 1990, MATLAB Macros for Polynomial $H_{\infty}$ Control System Optimization, Memorandum 881, Faculty of Maths, University of Twente, The Netherlands.

47. Bambang R., Shimemura E. and Uchida K., 1990, The discrete-time $H_{2} / H_{\infty}$ control synthesis: state feedback case, Korean Automatic Control Conf., 90 KACC, 10, 858-863.

48. Zhou K., Doyle, J., Glover K. and Bodenheimer B., 1990, Mixed $H_{2}$ and $H_{\infty}$ control, ACC Conf. Proc., San Diego, California, 2502-2507.

49. Hvostov H.S., 1990, On the structure of $H_{\infty}$ controllers, ACC Conf. Proc., San Diego, California, 2484-2489.

50. Rotea M.A. and Khargonekar P.P., 1991, $H_{2}$ optimal control with an $H_{\infty}$ constraint the state feedback case, Automatica, 27, (2), 307-316.

51. Rotea M.A. and Khargonekar P.P., 1990, Simultaneous $H_{2} \mathrm{I} H_{\infty}$ optimal control with state feedback, ACC Conf. Proc., San Diego, California, 2380-2384.

52. Steinbuch H., and Bosgra O.H., 1990, Necessary conditions for static and fixed order dynamic mixed $H_{2} / H_{\infty}$ optimal control, Selected Topics in Identification Modelling and Control, 2 , $17-23$.

53. Sparks A.G., Yeh H.H. and Banda S.S., 1990, Mixed $\mathrm{H}_{2}$ and $H_{\infty}$ optimal robust control design, Optimal Control Applications and Methods, I 1, 307-325.

54. Zhou K., Doyle J., Glover K. and Bodenheimer B., 1990, ACC Conf. Proc., San Deigo, California, 2502-2507.

55. Banda S.S., Yeh H.H. and Heise S.A., 1991, Robust control of uncertain systems with combined $H_{\infty}$ and $L Q G$ optimization, Int. J. Systems Sci., 22, (1), 85-96.

56. Grimble M.J., 1984, Implicit and explicit $L Q G$ self-tuning controllers, Automatica, 20, (5), 661-669.

57. Grimble M.J., 1986, Controllers for $L Q G$ self-tuning applications with coloured measurement noise and dynamic costing, Proc. IEE, Pt.D., 133, (1), 19-29.

58. Liu G.P. and Zakian V., 1990, Sup regulators, Proc. 29th Conf. on Decision and Control, Honolulu, Hawaii, 2145-2146.

59. Liu G.P., 1992, Mean regulators for discrete systems, IEE Proceedings - D, 139, (1), $67-71$. 
60. Liu G.P., 1992, Disturbance spaces and sup regulators and discrete time, Systems and Control Letters, 18, 33-38.

61. Lin S.F. and Chen C.H., 1992, Adaptive indirect weighted D-step-ahead control subject to an input amplitude constraint, Proc. American Control Conf., 2715-2719.

62. Pacini P.J. and Kosko B., 1992, Adaptive fuzzy systems for target tracking, Intelligent Systems Engineering, IEE, 1, (1), 3-21.

63. Desoer C.A. and Vidyasagar M., 1975, Feedback Systems: Input-output Properties, Academic Press, San Francisco.

64. Raven F.H., 1966, Mathematics of Engineering Systems, McGraw Hill, London.

65. Vidyasagar M., 1985, Control System Synthesis: A Factorization Approach, the MIT Press, Cambridge, Massachusetts.

66. Hosoda M., Kuribayashi T., Hirao F. and Takenaka K., 1991, Coil transfer expert systems for a hot strip mill finishing line, IFAC Expert Systems in Mineral and Metal Processing, Espoo, Finland, 89-94.

67. Lassila O., Mattila P., Pesonen L., Syrjanen M. and Tonna S., 1991, Applying knowledge-based techniques to the scheduling of steel rolling, IFAC Expert Systems in Mineral and Metal Processing, Espoo, Finland, 103-108.

68. Yaesh I. and Shaked U., 1992, $H_{\infty}$ optimal one-step-ahead output feedback control of discretetime systems, IEEE Trans. Auto. Control, 37, (8), 1245-1250.

69. Xie L. and de Souza C.E., 1992, Robust $H_{\infty}$ control for linear systems with norm-bounded time-varying uncertainty, IEEE Trans. Auto. Control, 37, (8), 1188-1191.

70. Zhou K., 1992, Comparison between $H_{2}$ and $H_{\infty}$ controllers, IEEE Trans. Auto. Control, 37, (8), 1261-1265.

71. Frank P.M., 1991, Enhancement of robustness in observer-based fault detection, IFAC Fault Detection, Supervision and Safety for Technical Processes, Baden-Baden, Germany, R. Isermann and B. Freyermuth (eds), 99-111.

72. Ding X. and Frank P.M., 1991, Frequency domain approach and threshold selector for robust model-based fault detection and isolation, IFAC Fault Detection, Supervision and Safety for Technical Processes, Baden-Baden, Germany, R. Isermann and B. Freyermuth (eds), 271-276.

73. De-kun Y., 1990, LQG self-tuning controller for coke oven battery heating, Research Report, Xinjiang Institute of Physics, Chinese Academy of Sciences.

74. Carr S.A., Anderson G., Grimble M.J. and Ringwood J., 1988, An $L Q G$ approach to self-tuning control with applications to robotics In: Robot Control, Theory and Applications, K. Warwick and A. Pugh (eds), Peter Peregrinus for IEE Control Eng., Series, 18-36.

75. Tse J., Bentsman J. and Miller N., 1992, Minimax predictive control, Proc. of 31st CDC, Tucson, Arizona, 2165-2170.

76. Forrest S.W., Grimble M.J. and Johnson M.A., 1992, On $L Q G$ self-tuning control Implementation and application, IEE Trans. Measurement and Control, 14, (5), 243-253.

77. Vardulakis A.I.G., 1991, Linear Multivariable Control, Algebraic Analysis and Synthesis Methods, John Wiley, Chichester.

78. Grimble M.J., 2001, Industrial Control Systems design, John Wiley, Chichester, ISBN 0471 492256.

79. Grimble M.J., 2000, Restricted structure $L Q G$ optimal control for continuous-time systems, IEE Proc. Control. Theory Appl., 161, 147, (2), 185-195.

80. Yukitomo M., Yutaka I., Hino S., Takahashi K. and Nagata K., 1998, A new PID controller tuning systems and its application to a flue gas temperature control in a gas turbine power plant, IEEE Conf. on Control Applications, Trieste, Italy, 2 (1-4), 1373-1377.

81. Grimble M.J., and Martin P., 2001, Adaptive control using controllers of restricted structure, IFAC Workshop on Periodic Control Systems, Lake Como, Italy.

82. Grimble M.J. and M.A. Johnson, 1988, Optimal Multivariable Control and Estimation Theory: Theory and Applications, Vol I and II, John Wiley, London. 
83. Nekoogar F. and Moriarty G., 1999, Digital Control Using Digital Signal Processing, PrenticeHall, New Jersey.

84. Patton R.J., Frank P.M. and Clark R.N., (eds), 2000, Issues of Fault Diagnosis for Dynamic Systems, Springer-Verlag, London, ISBN 3-540-19968-3.

85. Tadeo F., and Grimble M.J., 2002, Controller design using linear programming for systems with constraints, Part 3, Design examples, Computing and Control Engineering Journal, 89-93.

86. Grimble M.J., 1999, Restricted structure feedforward stochastic optimal control, CDC 99, Phoenix, Arizona.

87. Grimble M.J., 2000, Restricted structure linear estimators for multiple model and adaptive systems, SYSID 2000, Santa Barbara, California.

88. Grimble M.J. 2000, Restricted structure optimal linear pseudo-state filtering for discrete-time systems, American Control Conference, Chicago.

89. Harris T., 1989, Assessment of closed-loop performance, Canadian J. Chemical Eng., 67, 856861.

90. Desborough L.D. and Harris T.J., 1992, Performance assessment measures for univariate feedback control, Canadian J. of Chemical Eng., 70, 1186-1197.

91. Desborough L.D. and Harris T.J., 1993, Performance assessment measures for univariate feedback/feedforward control, Canadian J. of Chemical Eng., 71, 605-615

92. Desborough L.D. and Harris T.J., 1994, Control performance assessment, Pulp and Paper Canada, 95 (11), 441-443.

93. Huang B. and Shah S.L., 1999, Performance Assessment of Control Loops, Springer-Verlag. London.

94. Thornhill, N.F., Oettinger M. and Fedenczuk P., 1999, Refinery-wide control loop performance assessment, Journal of Process Control, 9, 109-124.

95. Boyd, S., El Ghaoui L., Feron E. and Balakrishnan V., 1994, Linear Matrix Inequalities in Systems and Control Theory, SIAM.

96. Willems J.C., 1971, Least squares stationary optimal control and the algebraic Riccati equation, IEEE Trans. Auto. Control, AC-16, (6), 621-634.

97. Hangstrup M.E., 1998, Strategies for Industrial Multivariable Control - with Application to Power Plant Control, PhD Thesis, Aalborg University, Denmark.

98. Babuška R. and Verbruggen H., 2003, Neuro-fuzzy methods for nonlinear system identification, Annual Reviews in Control, 27, 73-78.

99. Pinto Castillo S.E. and Madrigal G., 2001, Adaptive fuzzy control system for the load regulation of a fossil Power Plant, CICINDI, Mexico.

100. Pinto Castillo S. E. 2001, Control System with Adaptive Fuzzy Decoupling for the Regulation of Load of a Fossil Power Plant, MSc Thesis, CENIDET, Cuernavaca - Morelos, Mexico.

101. Verdult V., 2002, Nonlinear System Identification-A State-Space Approach, Twente University Press, Netherlands.

102. Grimble M.J., 2002, Controller performance benchmarking and tuning using generalised minimum variance control, Automatica, 38, 2111-2119.

103. Grimble M.J., 2004, Integral minimum variance control and benchmarking, Journal of Process Control, 14, 177-178.

104. Huang B., 2002, Minimum variance control and performance assessment of time-variant processes, Journal of Process Control, 12, 707-719.

105. Çağlayan N., Karacan S., Hapoğlu H. and Alpbaz M., 1997, Application of optimal adaptive control based on generalized minimum variance to a packed distillation column, Comp. Chemical Eng., 21, s607-s612.

106. Karagöz A. R., Hapoğlu H. and Alpbaz M., 2000, Generalized minimum variance control of optimal temperature profiles in a polystyrene polymerization reactor, Chemical Engineering and Processing, 39, 253-256. 
